PALABRAS CLAVE

Juventud

Empleo de los jóvenes

Mercado de trabajo

Ingresos

Desempleo

Medición

Evaluación

Aspectos sociales

Recursos humanos

Igualdad de género

Creación de empleos

Estadísticas del trabajo

América Latina

Jürgen Weller

Oficial de Asuntos Económicos

Unidad de Análisis Macroeconómico,

División de Desarrollo Económico,

CEPAL

œ jurgen.weller@cepal.org
REVISTA DE LA CEPAL 92 - AGOSTO 2007

\section{La inserción laboral de los jóvenes: características, tensiones y desafíos}

\author{
Jürgen Weller
}

$\mathrm{L}$

as debilidades de la inserción laboral de los jóvenes afectan no solamente su propio bienestar, sino también algunos elementos clave del desarrollo socioeconómico en general. Este artículo examina las circunstancias, orígenes y consecuencias de estas debilidades y revisa la información estadística sobre la evolución reciente de las variables laborales juveniles. Las cifras muestran que la situación laboral de los jóvenes empeoró en términos absolutos, en sintonía con el deterioro de los mercados laborales en general, y que, contrariamente a ciertas expectativas, tampoco mejoró en términos relativos. Se observa también una gran heterogeneidad de condiciones laborales, según el nivel educativo, el género y las características del hogar, entre otras cosas. El artículo identifica asimismo una serie de tensiones entre la subjetividad de los jóvenes y la realidad del mercado de trabajo, y pasa revista a opciones para mejorar la inserción laboral juvenil en torno a los temas de empleabilidad, equidad de género, espíritu emprendedor y creación de empleo. 


\section{I}

\section{Introducción}

Desde el inicio de la era moderna, la inserción laboral de los jóvenes es el elemento clave para pasar a la vida adulta, dado que los ingresos propios generan la base material para disminuir y luego eliminar la dependencia económica respecto de los padres y establecer un hogar propio. ${ }^{1}$ Siendo que la sociedad suele valorar a los individuos según su contribución al desarrollo material, cultural o espiritual, el trabajo también brinda integridad social y conlleva legitimidad y reconocimiento social. La inserción laboral es un ámbito de desarrollo interpersonal que facilita los contactos y la incorporación a redes, a la vez que permite participar en acciones colectivas. En resumen, el trabajo se puede interpretar como un eje de la integración social, fuente de sentido para la vida personal, espacio para la participación ciudadana y motor del progreso material (CEPAL/OIJ, 2003, p. 21).

Si bien los cambios económicos, sociales y culturales de las últimas décadas han relativizado — sobre todo desde la perspectiva propia de muchos jóvenes-el papel del trabajo en relación con otras actividades y aspectos de la vida, y se ha prolongado el tránsito de la juventud a la adultez (Hopenhayn, 2004), la inserción laboral sigue siendo un factor decisivo en el sentido descrito, en su calidad de pauta cultural preponderante, y también como aspiración individual de la inmensa mayoría de los jóvenes.
Sin embargo, recientemente las condiciones económicas y laborales no han favorecido la inserción de los jóvenes de la región en el mundo del trabajo. De hecho, preocupa la evolución de los mercados de trabajo de América Latina en los años 1990 y la primera mitad de los años 2000. En numerosas encuestas de opinión se indica que el desempleo y otras deficiencias laborales se hallan entre los problemas que más inquietan a la población latinoamericana y es rara la campaña electoral en que no se anuncia una significativa generación de empleos como una de sus metas.

En este artículo se examinan las características, tensiones y desafíos de la inserción laboral de la juventud latinoamericana. ${ }^{2}$ En la sección II se presenta el contexto de las debilidades de esta inserción, sobre todo el desempeño macroeconómico de América Latina y las transformaciones estructurales de los mercados, y se resume el debate sobre las causas y consecuencias de estos problemas. En la sección III se ilustra la evolución reciente de la situación laboral de los jóvenes de la región. En la sección IV se incorporan elementos de subjetividad, al analizar los contrastes entre la realidad de los mercados de trabajo latinoamericanos y las características, aspiraciones y expectativas de los jóvenes. En la sección V, por último, se analizan los desafíos que es necesario encarar para mejorar la inserción laboral de los jóvenes en América Latina.

\section{II \\ Circunstancias, orígenes y consecuencias de los problemas de inserción laboral de los jóvenes}

En el contexto de un crecimiento económico modesto y volátil, la generación de empleo asalariado - sobre todo formal - fue débil y la tasa de desempleo regional aumentó de un 7,5\% a un 11,0\% entre 1990 y 2003, hasta que la reactivación económica de los años posteriores

\footnotetext{
${ }^{1}$ Para facilitar la fluidez de la lectura, en este artículo la expresión "los jóvenes" abarca a los jóvenes de ambos sexos, salvo que se indique otra cosa.
}

permitió que bajara a un dígito, pero sin llegar al nivel de 1990 (CEPAL, 2006). A la vez, se extendió el sector

\footnotetext{
${ }^{2}$ Este trabajo se basa principalmente en los resultados de un proyecto llevado a cabo en la CEPAL con la cooperación de la Sociedad Alemana de Cooperación Técnica (GTZ) y el financiamiento del Ministerio de Cooperación Económica de Alemania. Véase más detalles en Weller (2006a), así como en las compilaciones de estudios nacionales de Carranza (2006), Chacaltana (2006a), Charlín y Weller (2006) y Martínez Valle (2006).
} 
informal y se incrementó la precariedad de las condiciones laborales. Como es lógico, este empeoramiento de la situación del mercado laboral también afectó a los jóvenes, lo que se ha reflejado, por ejemplo, en el alza de la tasa de desempleo juvenil (véase la sección III).

Aparte de los vaivenes de la coyuntura económica, existen procesos más bien estructurales que afectan a los mercados de trabajo y, en particular, a la inserción laboral juvenil. Entre ellos cabe resaltar las recientes dinámicas de la oferta y la demanda laboral, los cambios en el funcionamiento de los mercados de trabajo, así como las transformaciones socioeconómicas más allá de estos mercados.

Por el lado de la oferta laboral, destacan la reducción del crecimiento demográfico, el aumento del nivel educativo de las nuevas generaciones y la progresiva integración laboral de las mujeres jóvenes. Todas estas tendencias pueden favorecer una inserción laboral más productiva y equitativa de las nuevas generaciones.

Por el lado de la demanda, habría que resaltar la creciente integración comercial y financiera que, en forma directa o indirecta, intensifica la competencia en los mercados. Las empresas pueden responder de diferente manera a la presión de una competitividad siempre en alza; una de las respuestas — que probablemente tendrá una creciente gravitación - es la de incorporar nuevas tecnologías y nuevos procesos organizativos. Tal respuesta tiende a acrecentar la demanda relativa de mano de obra calificada y flexible, lo que también favorece la inserción laboral juvenil más que la de los adultos. Para estos últimos es más difícil enfrentar los cambios en las pautas productivas y tecnológicas. ${ }^{3}$

La aceleración del cambio en los mercados, como consecuencia de la integración global y los avances tecnológicos, afecta también al funcionamiento del mercado de trabajo. A veces facilitada por reformas legales, la inestabilidad laboral tendió a aumentar. Sin embargo, hasta ahora pareciera que en la mayoría de los países no ha habido transformaciones generalizadas de las relaciones contractuales, sino más bien cambios "en el margen". En efecto, mientras en el sector formal sigue predominando el contrato de plazo indeterminado, las nuevas contrataciones se caracterizan con mayor frecuencia por ser más inestables y ofrecer condiciones laborales deterioradas, lo que afecta sobre todo a los jóvenes.

\footnotetext{
${ }^{3}$ Véase el análisis correspondiente a los países avanzados en Blanchflower y Freeman (2000).
}

Otro elemento que complica la inserción laboral juvenil es la marcada (y creciente) segmentación socioeconómica, reflejo y origen de la elevada desigualdad en América Latina. En efecto, el trasfondo familiar determina en gran medida las perspectivas laborales de los jóvenes, pues influye en las oportunidades de acumular capital humano (acceso a educación y capacitación de buena calidad), capital social (relaciones sociales basadas en la confianza, la cooperación y la reciprocidad) y capital cultural (manejo de los códigos establecidos por la cultura dominante). La situación, los problemas y las perspectivas de los jóvenes, por lo tanto, son heterogéneas.

Se puede afirmar entonces que la situación laboral de los jóvenes latinoamericanos es crítica, dinámica y segmentada (CEPAL/OIJ, 2003). No obstante, conviene destacar que muchos de estos fenómenos no son específicamente latinoamericanos. De hecho, son muchos los organismos internacionales que en los últimos años han examinado los problemas de la inserción laboral de los jóvenes para buscar maneras de mejorarla. ${ }^{4} \mathrm{La}$ generación de empleo juvenil digno y productivo se ha definido como una de las metas en el marco de los objetivos de desarrollo del Milenio. ${ }^{5}$ En parte, esta renovada preocupación fue una reacción ante la ingrata sorpresa de comprobar que los indicadores de la inserción laboral de los jóvenes no mejoraban, a pesar de que algunas de las transformaciones en curso mencionadas más bien los favorecerían. De hecho, al observar en 2007 los avances de los diferentes indicadores en relación con los objetivos de desarrollo del Milenio, en pocos de ellos se registra un desempeño tan deficiente como en el empleo y desempleo juveniles. ${ }^{6}$

En el debate académico y político se han abordado varias explicaciones de las dificultades que enfrenta la

\footnotetext{
${ }^{4}$ Por ejemplo, véase OCDE (2002, cap. 1), Naciones Unidas (2004, cap. 3), OIT (2006) y Banco Mundial (2007, cap. 4).

${ }^{5}$ Se trata de la meta 16 dentro del objetivo 8 ("Fomentar una asociación mundial para el desarrollo"), que era la siguiente: "En cooperación con los países en desarrollo, elaborar y aplicar estrategias que proporcionen a los jóvenes un trabajo digno y productivo". Al respecto, se definió la tasa de desempleo de los jóvenes de 15 a 24 años, por sexo y total, como indicador clave (número 45) (http://www.un.org/ spanish/. millenniumgoals/\#, última visita:19.2.2007). Posteriormente se introdujo una meta de empleo productivo y trabajo decente para todos, "incluyendo mujeres y jóvenes", y se eliminó al mismo tiempo la citada meta 16 .

${ }^{6}$ La única región de la cual se esperaba que el año 2006 cumpliera la meta de empleo juvenil era Oceanía. En la mayoría de las regiones hubo un estancamiento o un empeoramiento (http://unstats.un.org/unsd/ mdg/Resources/Static/Products/Progress2006/MDGProgressChart2006. pdf, última visita 19.2.2007).
} 
inserción laboral juvenil. ${ }^{7}$ En cuanto a la oferta, destaca la hipótesis de que los sistemas educativos y de capacitación no preparan adecuadamente a los jóvenes para el mundo laboral. Como ya se dijo, las empresas - principales demandantes de mano de obra- enfrentan acelerados cambios económicos y tecnológicos. Para hacerles frente, muchas tienden a elevar y modificar sus requisitos en relación con las calificaciones de su fuerza laboral, tanto respecto de las habilidades y conocimientos técnicos y profesionales (hard skills) como de las competencias sociales y metodológicas, sobre todo las capacidades de comunicación, trabajo en equipo y solución de problemas (soft skills).

Frente a esta demanda creciente y dinámica, los sistemas de educación y capacitación adolecen de escasez de recursos, desconexión del mundo del trabajo y, por ende, ignorancia de las características de la demanda, así como también de una limitada capacidad de ajuste. Además, como es frecuente que exista incertidumbre sobre las futuras características de la demanda, las señales provenientes del mundo laboral no son claras, lo que obviamente dificulta las posibilidades de que se ajuste la oferta de educación y capacitación. Por lo tanto, los jóvenes saldrían de estos sistemas sin la preparación adecuada y desconociendo las características del mundo del trabajo; a su vez, las empresas tendrían reticencia a contratarlos. Además, contrariamente a lo que a veces se plantea, se puede argumentar que los profundos cambios tecnológicos y organizativos no eliminan del todo el valor de la experiencia. En consecuencia, aunque los jóvenes tengan habilidades específicas - por ejemplo, en nuevas tecnologías - sus ventajas potenciales frente a las personas de más edad se matizan debido a otras debilidades relativas.

Mientras que este primer grupo de problemas de inserción surge de la precariedad de la oferta para ajustarse a las características de la demanda, un segundo grupo surge de la demanda misma. En lo que toca a la magnitud de la demanda, cabe destacar que, en un enfriamiento económico, lo primero que las empresas hacen en sus políticas de recursos humanos es dejar de contratar, lo que obviamente afecta más a los jóvenes - representados en exceso entre los buscadores de empleo-que a otras personas. ${ }^{8}$ Cuando la crisis se profundiza y las

\footnotetext{
${ }^{7}$ Véase más detalles en Diez de Medina (2001), O’Higgins (2001), Fawcett (2002), Tokman (2003), Weller (2003) y Cacciamali (2005).

${ }^{8}$ Por ejemplo, el promedio de las cifras de Chile, Ecuador, El Salvador, Paraguay y Perú a inicios de la década del 2000 indica que un 52,7\% de los cesantes y un $84,6 \%$ de quienes buscaban trabajo por primera vez
}

empresas empiezan a reducir su personal, nuevamente los jóvenes suelen ser los más afectados, ya que por su menor antigüedad y debido a razones sociales (protección de los jefes de hogar) y económicas (mantención de la fuerza laboral experimentada, menores costos de despido) son los primeros en perder el empleo. Por lo tanto, en situaciones de gran volatilidad económica -características de América Latina durante las últimas décadas- el entorno se hace muy desfavorable para la inserción laboral de los jóvenes.

En el mercado de trabajo existe un problema de información incompleta: entre los jóvenes, respecto al mundo laboral en general y a determinadas empresas en particular; y entre las empresas, respecto a la juventud en general y a ciertos jóvenes en particular. La falta de transparencia de los procesos de intermediación, los prejuicios por ambas partes y las prácticas discriminatorias, pueden hacer aún más ineficiente y prolongado el proceso de ajuste entre los requisitos de las empresas y las aspiraciones de los jóvenes, así como profundizar desigualdades.

Además, regulaciones tales como un alto salario mínimo, que imponen un mayor costo a la contratación de jóvenes cuya productividad relativa es menor al estar limitada por su falta de experiencia, pueden reducir el nivel de empleo juvenil. Otras regulaciones, orientadas a proteger a los trabajadores que tienen empleo (insiders), tenderían a bloquear el acceso a los puestos de trabajo de los sin empleo (outsiders), entre ellos, los jóvenes.

Finalmente, la estructura productiva y ciertas características de la institucionalidad laboral restringen las posibilidades de una inserción ascendente en el mercado de trabajo. Un segmento importante de las economías de la región se caracteriza por una baja productividad, bajos niveles tecnológicos y el incumplimiento de muchas regulaciones, entre ellas las laborales. Un empleo en este segmento no solo implica condiciones laborales precarias, sino además que la experiencia laboral adquirida en él no es mayormente reconocida por el mercado, de manera que el premio salarial por este tipo de experiencia es bajo. Por otra parte, el abuso de ciertos mecanismos contractuales y el incumplimiento de las leyes laborales también pueden darse en un contexto más "formal", lo que empeora las características de la inserción laboral juvenil y limita sus futuras perspectivas.

La literatura especializada señala que hay alguna influencia de todos estos factores - muchos, por cierto,

eran jóvenes de 15 a 29 años (cálculo propio basado en tabulaciones especiales de las encuestas de hogares de los respectivos países). 
relacionados entre sí-, pero con importantes disparidades en su grado de incidencia (Weller, 2003). Como veremos más adelante, la heterogeneidad de los grupos etarios impide hacer generalizaciones, de modo que en el análisis de los procesos de inserción laboral juvenil y en las propuestas para mejorarlos es preciso tomar en cuenta las grandes diferencias de capital humano, social y cultural. Evidentemente, la definición de la juventud a partir de la edad biológica es muy limitada, pero en los estudios que trabajan con fuentes estadísticas no se tiene más alternativa que utilizarla. Por lo tanto, en este artículo se entiende por juventud las personas de 15 a 29 años.

Ahora bien, el hecho de que en los indicadores laborales se registren peores niveles para los jóvenes que para los adultos no sería por sí solo preocupante. Sin embargo, un desempleo juvenil alto y prolongado, así como una inserción de mala calidad e inferior a los niveles de educación y las habilidades adquiridas, tienen efectos negativos tanto económicos como sociales:

- El mal aprovechamiento del capital humano generado mediante el apoyo de la inversión social limita el crecimiento económico y, por consiguiente, el bienestar de las sociedades en su conjunto.

- Una débil acumulación de experiencia laboral incide negativamente en los ingresos laborales futuros de los jóvenes, así como en su jubilación, sobre todo en sistemas de capitalización individual.

- Una precaria inserción laboral dificulta y posterga la formación de hogares propios de los jóvenes, prologándose su dependencia respecto de los padres y la carga financiera que esto implica. Por lo tanto, también se reducen los ingresos netos presentes y —debido al impacto negativo en su capacidad de ahorro- los ingresos futuros de los padres.

- La inserción laboral precaria, temprana o tardía, relacionada frecuentemente con altos niveles de deserción o expulsión del sistema escolar, afecta especialmente a los jóvenes procedentes de hogares pobres, por lo que no se aprovecha el potencial aporte de una actividad laboral para que estos jóvenes salgan de esa situación. De esta manera se refuerza la transmisión intergeneracional de la pobreza.

- Un desfase entre las características de la educación y de la demanda laboral tiende a obstaculizar la movilidad social, con lo que se agravan los problemas estructurales de mala distribución del ingreso, perpetuando la inequidad de la distribución de los ingresos en la región.

- La precariedad de la inserción laboral obstaculiza la integración social de los jóvenes, quienes no se ven reconocidos en sus derechos ciudadanos; esto desalienta su participación en otros ámbitos de la institucionalidad vigente y tiende a fomentar en ellos actitudes de confrontación.

- Los jóvenes con inserción laboral precaria son una parte importante de la población en riesgo y enfrentan problemas de adaptación y marginación social.

\section{III}

\section{La evolución reciente de la inserción laboral de los jóvenes en América Latina}

Esta sección examina las características de la inserción laboral juvenil en América Latina, y los cambios ocurridos entre los inicios de los años 1990 y los primeros años de la década del $2000 .{ }^{9}$ Cabe recordar que estos datos reflejan

\footnotetext{
${ }^{9}$ Esta sección se basa principalmente en el procesamiento de encuestas de hogares de 17 países, realizado por Carlos Daroch. Dado que no se dispone de información estadística de los mismos años para todos los países, se ha procurado procesar la información disponible más reciente (para la mayoría de los países corresponde a 2003 o 2004) y los datos para un año de los inicios de la década de 1990 o, en el caso de países que no disponen de esta información, para un año de la primera
}

un crecimiento económico mediocre (2,9\% por año entre 1990 y 2004, lo que corresponde a un crecimiento anual de 1,2\% del PIB per cápita) y, de manera correspondiente, un desempeño poco satisfactorio de los mercados de trabajo. Como se ve a continuación, la inserción laboral juvenil no escapó a estas tendencias.

mitad de dicha década (véase más detalles al respecto en la nota del cuadro 1). Para poder identificar tendencias regionales se calcularon promedios simples de los países con información comparable. Se ha denominado "período reciente" aquel entre los inicios de la década de 1990 y los primeros años de la década del 2000. 


\section{Evolución de la actividad y la inactividad juvenil}

En América Latina, dos tendencias resaltan en el período reciente con respecto a la participación de los jóvenes en el mercado de trabajo. Una es la moderada caída de la tasa de participación ${ }^{10}$ de los hombres; la segunda, el marcado aumento de la tasa de participación de las mujeres. Como consecuencia, se redujo la brecha de participación entre hombres y mujeres (cuadro 1).

Como saldo de las dos tendencias opuestas señaladas, en el conjunto de los jóvenes se registró un leve aumento de la participación laboral (salvo en el grupo más joven). La caída de la tasa de participación de los hombres jóvenes se debe, sobre todo, a su contracción en el grupo de menor edad (15 a 19 años). Una tendencia fundamental al respecto es su mayor permanencia en el sistema educativo, ya que aumentó la proporción de estudiantes, sobre todo en este grupo etario (cuadro 2). A la vez, entre los hombres jóvenes bajó levemente la participación de los "Otros inactivos", que es el grupo que contiene el principal contingente de jóvenes en mayor riesgo de exclusión y marginación. En este contexto, la merma de la tasa de participación de los jóvenes es una tendencia positiva. Sin embargo, todavía persisten problemas: por ejemplo, la elevada participación laboral de los jóvenes entre 15 y 19 años, edad en que debe-

${ }^{10}$ La tasa de participación representa la proporción de la población en edad de trabajar que se encuentra ocupada o busca trabajo. rían, en su gran mayoría, asistir a los establecimientos educativos, y el hecho de que más del $5 \%$ de este grupo etario pertenece a "Otros inactivos".

La mayor participación laboral de las mujeres jóvenes no condujo a caída alguna de su asistencia al sistema escolar: en todos los grupos etarios aumentó la proporción de estudiantes mujeres, y en todos ellos la asistencia escolar de las mujeres superó la de los hombres de la misma edad. Es de suponer que a ello contribuyó la conciencia de que para mujeres de bajo nivel educativo existen menos oportunidades de empleo que para los hombres de igual condición, por lo que aquellas harían un esfuerzo mayor por calificarse a fin de mejorar sus opciones de acceder a empleos de mejor calidad.

En contraste, bajó marcadamente la proporción de las jóvenes que se desempeña en oficios domésticos, mientras que la proporción de aquellas incluidas en "Otros inactivos" mostró pocos cambios. El incremento paralelo de la asistencia al sistema escolar y de la inserción laboral puede considerarse como otra tendencia positiva. Nuevamente, eso no significa que los problemas de inactividad laboral estén superados, ya que todavía más de una de cada cinco jóvenes entre 15 y 19 años se ocupa de oficios domésticos o se encuentra entre los "Otros inactivos", lo que restringe severamente las condiciones de una futura inserción en el mercado de trabajo.

La mayor asistencia al sistema educativo se manifiesta no solo en el aumento de la proporción de estudiantes como porcentaje de los grupos etarios correspondientes, sino también en la mayor proporción de jóvenes ocupados

CUADRO 1

América Latina (17 países): tasa de participación por grupos etarios y sexo, entre alrededor de 1990 y alrededor de 2003/2004a

(En promedios simples)

\begin{tabular}{|c|c|c|c|c|c|c|}
\hline \multirow{2}{*}{ Grupo de edad } & \multicolumn{3}{|c|}{ Alrededor de 1990} & \multicolumn{3}{|c|}{ Alrededor de 2003/2004 } \\
\hline & Ambos & Hombres & Mujeres & Ambos & Hombres & Mujeres \\
\hline $15-19$ & 38,9 & 52,4 & 25,5 & 38,2 & 48,9 & 27,5 \\
\hline $20-24$ & 64,2 & 83,3 & 46,1 & 68,9 & 84,0 & 54,3 \\
\hline $25-29$ & 71,5 & 94,8 & 50,4 & 77,2 & 93,9 & 61,8 \\
\hline $15-29$ & 56,5 & 74,4 & 39,7 & 59,2 & 72,2 & 46,5 \\
\hline $30-64$ & 68,3 & 92,8 & 45,9 & 74,8 & 93,1 & 58,3 \\
\hline
\end{tabular}

Fuente: CEPAL, sobre la base de tabulaciones especiales de las encuestas de hogares de los respectivos países.

a La cobertura es el total nacional para Brasil, Chile, Colombia, Costa Rica, El Salvador, Guatemala, Honduras, México, Nicaragua, Panamá, Perú, República Dominicana y Rep. Bolivariana de Venezuela; el total urbano para Bolivia, Ecuador y Uruguay, y el Gran Buenos Aires para Argentina. Para el período alrededor de 1990, los años son 1989 para Bolivia, Guatemala y México; 1990 para Argentina, Brasil, Chile, Costa Rica, Honduras, Uruguay y Rep. Bolivariana de Venezuela; 1991 para Colombia y Panamá; 1993 para Nicaragua; 1995 para El Salvador, y 1997 para Ecuador, Perú y la República Dominicana. Para el período alrededor de 2003/2004, los años son 2001 para Nicaragua; 2002 para Bolivia; 2003 para Brasil, Chile, Honduras, Perú y Rep. Bolivariana de Venezuela; 2004 para Argentina, Colombia, Costa Rica, Ecuador, El Salvador, Guatemala, México, Panamá, la República Dominicana y Uruguay. 
(Porcentajes, en promedios simples)

\begin{tabular}{|c|c|c|c|c|c|c|}
\hline \multirow{2}{*}{ Grupo etario } & \multicolumn{3}{|c|}{ Alrededor de 1990} & \multicolumn{3}{|c|}{ Alrededor de 2003/2004 } \\
\hline & Ambos & Hombres & Mujeres & Ambos & Hombres & Mujeres \\
\hline \multicolumn{7}{|l|}{ Estudiantes } \\
\hline $15-19$ & 44,9 & 42,4 & 47,3 & 48,9 & 46,4 & 51,5 \\
\hline $20-24$ & 12,3 & 11,9 & 12,7 & 13,5 & 12,4 & 14,5 \\
\hline $25-29$ & 2,7 & 2,4 & 2,9 & 2,9 & 2,7 & 3,2 \\
\hline \multicolumn{7}{|c|}{ Oficios domésticos } \\
\hline $15-19$ & 13,0 & 0,8 & 25,2 & 9,6 & 0,9 & 18,3 \\
\hline $20-24$ & 20,2 & 0,3 & 38,6 & 14,8 & 0,4 & 28,5 \\
\hline $25-29$ & 23,7 & 0,1 & 44,9 & 17,0 & 0,3 & 32,3 \\
\hline \multicolumn{7}{|c|}{ Otros inactivos } \\
\hline $15-19$ & 5,3 & 6,2 & 4,3 & 5,1 & 5,6 & 4,6 \\
\hline $20-24$ & 4,4 & 4,5 & 4,2 & 4,0 & 3,9 & 4,1 \\
\hline $25-29$ & 3,1 & 3,2 & 3,1 & 3,0 & 3,0 & 3,0 \\
\hline \multicolumn{7}{|c|}{ Total inactivos } \\
\hline $15-19$ & 63,1 & 49,3 & 76,8 & 63,6 & 53,0 & 74,3 \\
\hline $20-24$ & 36,8 & 16,7 & 55,5 & 32,2 & 16,8 & 47,1 \\
\hline $25-29$ & 29,5 & 5,8 & 50,9 & 22,9 & 5,9 & 38,5 \\
\hline
\end{tabular}

Fuente: CEPAL, sobre la base de tabulaciones especiales de las encuestas de hogares de los respectivos países.

${ }^{\text {a }}$ Por falta de datos, de la cobertura especificada en el cuadro 1, se excluye a Brasil y Perú.

y desocupados que asisten al sistema educativo. Este grupo es muy importante, pues alrededor de 2003/2004 abarca el $35,1 \%$ de los ocupados entre 15 y 19 años, el $20,8 \%$ del grupo de 20 a 24 años y el 12,2\% del grupo de 25 a 29 años, con tasas más altas entre las mujeres que entre los hombres. ${ }^{11}$ Las proporciones son parecidas entre los desocupados, de los cuales el $36,2 \%$, el $24,6 \%$ y el $13,7 \%$ de los tres grupos etarios, respectivamente, asisten al sistema educativo.

Finalmente, existe una clara diferencia entre hombres y mujeres jóvenes con respecto a su participación laboral, según el ingreso del hogar al que pertenecen (Weller, 2006b, p. 9). Específicamente, entre los hombres se observa una curva de U invertida, con las tasas más bajas en el primer y quinto quintil de ingresos, si bien las diferencias entre los quintiles son menores. ${ }^{12}$

\footnotetext{
${ }^{11}$ En el período reciente hubo un aumento generalizado de estos porcentajes. Alrededor de 1990, la proporción de los ocupados que también asistieron al sistema educativo fue, para los tres grupos etarios, de $26,6 \%, 14,9 \%$ y $7,7 \%$, respectivamente (calculada como promedio simple de 13 países).

${ }_{12}$ Alrededor de cinco puntos porcentuales entre la tasa más baja y la más alta.
}

En contraste, en el caso de las mujeres jóvenes hay una clara correlación positiva entre el nivel de ingreso del hogar y la participación laboral. En efecto, las jóvenes pertenecientes a los hogares más pobres (primer quintil) tienen una tasa de participación inferior en alrededor de 20 puntos porcentuales a la de las jóvenes de su misma edad en el quintil más rico.

\section{Las tendencias del empleo juvenil}

Entre inicios de los años 1990 y mediados de los años 2000 la tasa de ocupación ${ }^{13}$ de los jóvenes creció levemente, como resultado de la caída de la tasa de los hombres jóvenes y su moderado aumento en el caso de las jóvenes (cuadro 3).

La tasa de ocupación exhibe una mayor homogeneidad entre los grupos educativos de los hombres jóvenes - con las tasas más altas en los grupos de menor nivel educativo- y una mayor heterogeneidad entre los de las mujeres - con las tasas más altas en los grupos de

\footnotetext{
${ }^{13}$ La tasa de ocupación representa la proporción de la población en edad de trabajar que se encuentra ocupada.
} 
América Latina (16 países): tasa de ocupación de los jóvenes de 15 a 29 años de edad, según nivel educacional y sexo, entre alrededor de 1990 y alrededor de 2003/2004a

(En promedios simples)

\begin{tabular}{|c|c|c|c|c|c|c|}
\hline \multirow{2}{*}{ Años de estudio } & \multicolumn{3}{|c|}{ Alrededor de 1990} & \multicolumn{3}{|c|}{ Alrededor de 2003/2004 } \\
\hline & Ambos & Hombres & Mujeres & Ambos & Hombres & Mujeres \\
\hline 0 a 3 & 52,3 & 76,7 & 28,2 & 54,6 & 74,7 & 32,0 \\
\hline 4 a 6 & 55,2 & 77,1 & 32,9 & 56,7 & 76,4 & 35,4 \\
\hline 7 a 9 & 40,9 & 55,8 & 26,5 & 41,9 & 53,9 & 29,2 \\
\hline 10 a 12 & 48,0 & 59,6 & 38,0 & 48,6 & 59,0 & 39,2 \\
\hline 13 y más & 55,2 & 59,9 & 51,1 & 55,2 & 59,6 & 51,8 \\
\hline Total & 49,0 & 66,5 & 32,6 & 50,0 & 63,5 & 36,9 \\
\hline
\end{tabular}

Fuente: CEPAL, sobre la base de tabulaciones especiales de las encuestas de hogares de los países.

a Por falta de datos, de la cobertura especificada en el cuadro 1, se excluye Argentina.

CUADRO 4

América Latina (14 países): porcentaje de jóvenes de 15 a 29 años de edad en empleos de baja productividad, según nivel educativo, entre alrededor de 1990 y alrededor de 2003/2004ª (En promedios simples)

\begin{tabular}{|c|c|c|c|c|c|c|}
\hline \multirow{2}{*}{ Años de estudio } & \multicolumn{3}{|c|}{ Alrededor de 1990} & \multicolumn{3}{|c|}{ Alrededor de 2003/2004 } \\
\hline & Ambos & Hombres & Mujeres & Ambos & Hombres & Mujeres \\
\hline 0 a 3 & 68,4 & 64,1 & 77,0 & 72,3 & 66,9 & 83,8 \\
\hline 4 a 6 & 62,7 & 58,0 & 72,6 & 66,3 & 61,4 & 77,2 \\
\hline 7 a 9 & 51,0 & 47,2 & 59,0 & 58,5 & 53,2 & 68,8 \\
\hline 10 a 12 & 32,9 & 32,1 & 34,4 & 40,0 & 36,7 & 44,7 \\
\hline 13 y más & 15,7 & 16,4 & 15,2 & 19,3 & 19,3 & 19,5 \\
\hline Total & 49,9 & 48,3 & 51,6 & 51,4 & 49,6 & 55,1 \\
\hline
\end{tabular}

Fuente: CEPAL, sobre la base de tabulaciones especiales de las encuestas de hogares de los países.

a Por falta de datos, de la cobertura especificada en el cuadro 1, se excluyen Argentina, Colombia y México.

mayor nivel educativo. Mientras que en el caso de los hombres esta situación se debe, sobre todo, al hecho de que una parte de los jóvenes de niveles educativos medios y altos todavía está estudiando, en el caso de las mujeres refleja más que todo las limitaciones culturales y las pocas oportunidades de empleo para mujeres jóvenes de menor nivel educativo (provenientes de familias de bajo nivel de ingreso, sobre todo en zonas rurales).

Entre las mujeres jóvenes, el nivel de ocupación subió en todos los grupos educativos, con mayores alzas en los grupos educativos más bajos, lo que derivó en una leve reducción de la diferencia de la tasa de ocupación entre las mujeres de diferente nivel educativo. Debido a efectos de composición, la brecha de la tasa de ocupación entre hombres y mujeres jóvenes se redujo en forma relativamente pronunciada, de 34 puntos porcentuales a inicios de los años 1990 a menos de 27 puntos alrededor de 2003/2004, si bien se mantiene alta. ${ }^{14}$

Además, en el período reciente hubo un aumento generalizado del peso de los sectores de baja productividad en la estructura ocupacional, lo que refleja principalmente la debilidad de la demanda laboral de los sectores más productivos en un contexto de bajo crecimiento económico. ${ }^{15}$ Existe una correlación negativa fuerte entre el peso de los sectores de baja productividad y el nivel educativo de los jóvenes (cuadro 4). En efecto,

\footnotetext{
14 Tanto entre los hombres como entre las mujeres, se incrementa la proporción de los grupos educativos más altos, pero estos grupos presentan tasas de ocupación inferiores al promedio en el caso de los hombres y superiores al promedio en el caso de las mujeres.

15 Estos sectores se miden con las variables sustitutivas (proxy) de trabajadores por cuenta propia sin calificación profesional o técnica, asalariados de microempresas, trabajadores familiares no remunerados y empleadas domésticas.
} 
CUADRO 5

América Latina (17 países): tasa de desempleo, según sexo y grupos de edad, entre alrededor de 1999 y alrededor de 2003/2004 (En promedios simples)

\begin{tabular}{|c|c|c|c|c|c|c|}
\hline \multirow{2}{*}{ Grupo de edad } & \multicolumn{3}{|c|}{ Alrededor de 1990} & \multicolumn{3}{|c|}{ Alrededor de 2003/2004 } \\
\hline & Ambos & Hombres & Mujeres & Ambos & Hombres & Mujeres \\
\hline $15-19$ & 17,7 & 15,6 & 22,1 & 22,4 & 19,2 & 28,1 \\
\hline $20-24$ & 13,4 & 11,2 & 16,7 & 16,8 & 13,9 & 21,1 \\
\hline $25-29$ & 9,0 & 7,3 & 11,7 & 10,6 & 8,0 & 14,0 \\
\hline $15-29$ & 12,8 & 10,9 & 15,9 & 15,9 & 13,1 & 19,9 \\
\hline
\end{tabular}

Fuente: CEPAL, sobre la base de tabulaciones especiales de las encuestas de hogares de los países.

en los niveles educativos más bajos, la proporción de estos sectores triplica con creces la proporción correspondiente al nivel educativo más alto. ${ }^{16}$ Sin embargo, durante el período más reciente, el peso de los sectores de baja productividad se incrementó también en los grupos educativos medios y altos, lo que indicaría que en el contexto de un bajo dinamismo de las economías de la región y con el aumento del nivel educativo de los jóvenes que entran al mercado de trabajo, un número creciente de estos jóvenes con nivel de educación bueno no encuentra un empleo acorde con su formación.

Finalmente, los jóvenes suelen presentar una movilidad mucho mayor en su condición de actividad laboral, y sobre todo una mayor inestabilidad en el trabajo. En el caso de Chile, Henríquez y Uribe-Echevarría (2003, p. 93) encontraron que, a lo largo de seis trimestres seguidos, solo un $23,3 \%$ de los jóvenes que en algún momento formaron parte de la población económicamente activa estuvieron siempre ocupados, mientras el 34,3\% registró tránsitos entre la ocupación y la inactividad y el $42,4 \%$ tuvo por lo menos una experiencia de desempleo. ${ }^{17} \mathrm{En}$ comparación, en el grupo de 30 a 49 años las cifras reflejan una estabilidad mucho mayor: son de un $60,4 \%$, un $19,8 \%$ y un $19,8 \%$, respectivamente.

\section{Las tendencias del desempleo juvenil}

En América Latina, la tasa de desempleo juvenil duplica con creces la de los adultos - un 15,9\% comparado

\footnotetext{
${ }^{16}$ Un ejercicio probit mostró para los casos de Argentina, Costa Rica y Rep. Bolivariana de Venezuela que la probabilidad de inserción en sectores de baja productividad desciende con el nivel educativo (Weller, 2003, pp. 50-52). Además, la pertenencia a hogares pobres aumenta la probabilidad de inserción en estos sectores.

17 Véase en Chacaltana (2006, p. 189) los datos correspondientes a Perú.
}

con un 6,6\% alrededor de 2003/2004-, y la brecha entre jóvenes y adultos es parecida para hombres y mujeres. En el período reciente el desempleo aumentó para todos los grupos, de manera que el incremento del desempleo juvenil fue más el reflejo del deterioro general de los mercados de trabajo de la región que de aspectos específicos que afectaran a los jóvenes. Incluso, en términos relativos, el desempleo creció un poco más para los adultos, de manera que la brecha entre ellos y los jóvenes disminuyó levemente. ${ }^{18}$ Entre los jóvenes, la tasa de desempleo de las mujeres superó a la de los hombres en casi la mitad, sin que se observaran mayores cambios en el período reciente (cuadro 5).

Existe una marcada correlación negativa entre el nivel de desempleo de los jóvenes y los ingresos del hogar. A inicios de la década del 2000, en el primer quintil la tasa de desempleo juvenil alcanzó casi al $30 \%$, más del triple de la que registró el quintil quinto. En el período reciente, sin embargo, este último quintil experimentó el mayor aumento proporcional de la tasa de desempleo, en parte posiblemente como consecuencia del mayor "desempleo académico", o sea, el desempleo de los jóvenes con mayor nivel de educación. Por lo demás, la pertenencia a hogares acomodados permite períodos más prolongados de espera y búsqueda, sin mayores sacrificios del bienestar de los miembros del hogar (Weller, 2006b, p. 20).

Cabe destacar que no existen grandes diferencias en el tiempo de búsqueda de trabajo entre cesantes jóvenes $\mathrm{y}$ adultos, lo que indica que los primeros en general no

\footnotetext{
${ }^{18}$ En el grupo de 17 países con datos comparables para el período reciente, el desempleo subió de $12,8 \%$ a $16,1 \%$ entre los jóvenes, y de $4,8 \%$ a $7,0 \%$ entre los adultos, con lo que la tasa de los primeros superaba a la de los segundos en un $170 \%$ a inicios de los años 1990 , y en un $130 \%$ una década después.
} 
América Latina (16 países): ${ }^{a}$ relación entre cesantes recientes ${ }^{b}$ y ocupados, por grupo de edad y por sexo, alrededor de 2003/2004

(Porcentajes, en promedios simples)

\begin{tabular}{lcccr}
\hline & $\begin{array}{c}15-19 \\
\text { años }\end{array}$ & $\begin{array}{c}20-24 \\
\text { años }\end{array}$ & $\begin{array}{c}25-29 \\
\text { años }\end{array}$ & $\begin{array}{c}\text { 15-29-64 } \\
\text { años }\end{array}$ \\
\cline { 2 - 5 } Ambos sexos & 4,4 & 3,2 & 2,2 & 2,9 \\
Hombres & 4,3 & 2,9 & 1,9 & 2,7 \\
Mujeres & 4,7 & 3,8 & 2,6 & 1,2 \\
\hline
\end{tabular}

Fuente: CEPAL, sobre la base de tabulaciones especiales de las encuestas de hogares de los países.

a Por falta de datos, de la cobertura especificada en el cuadro 1, se excluye Brasil.

b Se refiere a cesantes que buscan empleo desde hace hasta un mes.

tienen más problemas de acceso al mercado de trabajo que los segundos, aunque hay grupos específicos que sí pueden enfrentar problemas mayores. ${ }^{19}$ Sin embargo, como ya se mencionó, entre las cohortes jóvenes se concentran los desempleados que buscan trabajo por primera vez, y a estos típicamente les es más difícil acceder al mercado laboral, lo que se traduce en tiempos de búsqueda más largos. Estos dos factores relacionados inciden en la mayor tasa de desempleo juvenil y subrayan la importancia del acceso de los jóvenes a un primer empleo, y en especial a un primer empleo en que puedan acumular experiencias que sean reconocidas posteriormente por el mercado laboral.

De todas maneras, una vez empezando a acumular experiencia, los problemas específicos de los jóvenes - en comparación con los adultos- se concentran en las características de los puestos disponibles, más que en el acceso mismo a tales puestos; esto se expresa en una mayor inestabilidad laboral de los jóvenes en comparación con los adultos, entre otros motivos porque un alto porcentaje de sus contratos son de corta duración (Fajnzylber y Reyes, 2005). El cuadro 6 indica esta mayor inestabilidad con una mayor tasa de salida desde la ocupación al desempleo. Se observa en el cuadro que la relación entre el número de cesantes que perdieron su empleo recientemente y el de los ocupados es claramente más alta para los jóvenes que para los adultos, así como para las mujeres que para los hombres. ${ }^{20}$

\footnotetext{
${ }^{19}$ Por ejemplo, las mujeres jóvenes registran tiempos de búsqueda más prolongados que sus pares masculinos, aunque con una brecha menor que la registrada entre mujeres y hombres adultos (Weller, 2003, p. 34).

${ }^{20}$ Además, los jóvenes típicamente muestran una mayor rotación entre el empleo y la inactividad; por ejemplo, respecto al caso peruano véase Chacaltana (2006b, p. 189).
}

En consecuencia, el hecho de que la tasa de desempleo de los jóvenes sea mayor que la de los adultos se debe principalmente a la concentración en los jóvenes de las personas que buscan empleo por primera vez, a los problemas de acceso de estos buscadores primerizos y a la mayor rotación entre el empleo y el desempleo o la inactividad laboral que caracteriza a los jóvenes en comparación con los adultos.

\section{Las tendencias de los ingresos laborales}

Existe una gran brecha entre los ingresos de los jóvenes y de los adultos, porque estos últimos reciben un "premio a la experiencia". Lógicamente, la brecha se reduce con el aumento de la edad (y la experiencia) de los jóvenes. En efecto, mientras los más jóvenes (de 15 a 19 años, con un ingreso medio de aproximadamente 1,5 veces la línea de pobreza) ganan en promedio un tercio de los ingresos medios de los adultos, los jóvenes de 20 a 24 años ganan más de la mitad (2,6 veces la línea de pobreza), y los jóvenes de 25 a 29 años más de las tres cuartas partes (3,5 veces la línea de pobreza) del ingreso medio de los adultos, quienes en promedio tienen un ingreso que corresponde a 4,6 veces la línea de pobreza (cuadro 7). ${ }^{21}$ Contrariamente a las expectativas de una mejora al menos relativa de la inserción laboral de los jóvenes, en el período reciente tanto los ingresos reales medidos en términos de la línea de pobreza como las brechas entre jóvenes y adultos se han mantenido bastante estables.

Se observa que la brecha salarial es claramente mayor para los hombres que para las mujeres jóvenes,

\footnotetext{
${ }^{21}$ Esta mejoría también se observa al seguir la evolución de los ingresos de una cohorte etaria (véase Weller, 2003, pp. 53-60).
} 
América Latina (16 países): ingreso laboral de los jóvenes en relación con la línea de pobreza y el ingreso medio del adulto correspondiente, por grupo de edad y por sexo, entre alrededor de 1990 y alrededor de $2002^{a}$ (En promedios simples)

\begin{tabular}{|c|c|c|c|c|c|c|}
\hline & \multicolumn{3}{|c|}{ Alrededor de 1990} & \multicolumn{3}{|c|}{ Alrededor de 2002} \\
\hline & $\begin{array}{c}\text { 15-19 } \\
\text { años }\end{array}$ & $\begin{array}{c}20-24 \\
\text { años }\end{array}$ & $\begin{array}{c}25-29 \\
\text { años }\end{array}$ & $\begin{array}{c}\text { 15-19 } \\
\text { años }\end{array}$ & $\begin{array}{c}20-24 \\
\text { años }\end{array}$ & $\begin{array}{c}25-29 \\
\text { años }\end{array}$ \\
\hline \multicolumn{7}{|c|}{ Ingreso en relación con la línea de pobreza } \\
\hline Ambos sexos & 1,5 & 2,6 & 3,5 & 1,5 & 2,6 & 3,5 \\
\hline Hombres & 1,6 & 2,8 & 3,9 & 1,6 & 2,8 & 3,9 \\
\hline Mujeres & 1,4 & 2,2 & 2,9 & 1,3 & 2,3 & 3,0 \\
\hline \multicolumn{7}{|c|}{ Ingreso en relación con el ingreso medio del adulto correspondiente (porcentajes) } \\
\hline Ambos sexos & 33,0 & 56,9 & 78,0 & 32,6 & 57,0 & 77,2 \\
\hline Hombres & 29,6 & 52,8 & 74,2 & 29,6 & 53,0 & 73,3 \\
\hline Mujeres & 43,6 & 70,9 & 92,1 & 38,7 & 66,2 & 86,7 \\
\hline
\end{tabular}

Fuente: CEPAL, sobre la base de tabulaciones especiales de las encuestas de hogares de los países.

a Para los años 2003/2004 se dispone de datos de ingresos solo para pocos países, por lo que se muestran los datos de alrededor de 2002.

lo que indica que las mujeres, a lo largo de su vida laboral, reciben un menor premio a la experiencia que los hombres, sea porque realmente en promedio acumulan menos experiencia, debido a sus trayectorias laborales más interrumpidas, o bien a prácticas discriminatorias en las remuneraciones, $\mathrm{o}$ a ambos factores.

La brecha salarial entre jóvenes y adultos suele ser mayor en los niveles educativos más altos y menor en los niveles educativos bajos. En parte, esto se debe a que la experiencia desempeña un papel preponderante en el caso de la mano de obra calificada, dado que en sus actividades existe mayor espacio para desarrollar habilidades adicionales que en las ocupaciones más sencillas. Hay que considerar que en estas últimas el esfuerzo físico es un componente relevante del desempeño. ${ }^{22}$

En este contexto llama la atención que en el período reciente, mientras la literatura especializada indica que en el conjunto del mercado de trabajo la brecha salarial entre los más calificados y los otros grupos educativos se ha ensanchado (BID, 2003), la evidencia es mixta para los diferentes subgrupos etarios de jóvenes. Específicamente, los ingresos relativos de jóvenes con 10 a 12 años de educación tendieron a empeorar y los más educados registraron un mejoramiento de sus

\footnotetext{
${ }^{22}$ Además, hay que tomar en cuenta que, dentro de cada grupo etario, las personas con menor nivel educativo tienen potencialmente un mayor número de años de experiencia laboral, debido a su inserción más temprana en el mercado de trabajo.
}

ingresos relativos en el grupo de 25 a 29 años, pero no en el de 20 a 24 años (cuadro 8). En cambio, entre los adultos el grupo de 10 a 12 años de educación, y sobre todo el grupo más educado, mejoraron sus ingresos en comparación con los otros grupos educativos.

Estos resultados contradicen la hipótesis ampliamente compartida de que los profundos cambios tecnológicos recientes han dado ventajas competitivas a los jóvenes más educados, con habilidades en nuevos campos tecnológicos, ya que estas habilidades son más difíciles de adquirir por los adultos que se formaron en el contexto de otros paradigmas tecnológicos. Respecto al grupo de 10 a 12 años de educación, esta evolución inesperada posiblemente se debe a que en el período reciente el aumento de la cobertura de la educación secundaria ha "devaluado" este logro de estudios, y los jóvenes con este nivel educativo que entraron en forma masiva al mercado de trabajo vieron caer sus ingresos relativos. Respecto al grupo más educado, dados los problemas para encontrar empleo en puestos de trabajo conforme a su nivel educativo (creciente "desempleo académico"), una parte de los nuevos entrantes al mercado laboral habría tenido que emplearse en puestos por debajo de su nivel de calificación, lo que afectaría negativamente los ingresos medios del grupo educativo correspondiente.

Existen importantes diferencias de ingresos entre hombres y mujeres jóvenes, tanto en su conjunto como para grupos educativos específicos. La brecha crece con el aumento de la edad, ya que, en promedio, el ingreso de 
América Latina (16 países): ingreso laboral respecto del ingreso medio de los ocupados con 10 a 12 años de estudio del grupo de edad correspondiente, por grupo de edad y según nivel educativo, entre alrededor de 1990 y alrededor del 2002 (En promedios simples)

\begin{tabular}{|c|c|c|c|c|c|c|c|c|}
\hline \multirow[b]{2}{*}{ Años de estudio } & \multicolumn{4}{|c|}{ Alrededor de 1990} & \multicolumn{4}{|c|}{ Alrededor del 2002} \\
\hline & $\begin{array}{l}15-19 \\
\text { años }\end{array}$ & $\begin{array}{l}20-24 \\
\text { años }\end{array}$ & $\begin{array}{c}25-29 \\
\text { años }\end{array}$ & $\begin{array}{c}30-64 \\
\text { años }\end{array}$ & $\begin{array}{l}15-19 \\
\text { años }\end{array}$ & $\begin{array}{c}20-24 \\
\text { años }\end{array}$ & $\begin{array}{c}25-29 \\
\text { años }\end{array}$ & $\begin{array}{c}\text { 30-64 } \\
\text { años }\end{array}$ \\
\hline Total & 74,0 & 86,4 & 89,6 & 82,2 & 89,9 & 92,3 & 100,0 & 95,9 \\
\hline 0-3 años & 61,6 & 58,9 & 55,6 & 50,4 & 84,4 & 68,6 & 58,6 & 50,1 \\
\hline 4-6 años & 72,2 & 80,2 & 67,3 & 66,3 & 87,4 & 79,0 & 73,5 & 64,7 \\
\hline 7-9 años & 77,2 & 82,0 & 83,0 & 79,3 & 87,4 & 86,0 & 84,3 & 75,9 \\
\hline 10-12 años & 100,0 & 100,0 & 100,0 & 100,0 & 100,0 & 100,0 & 100,0 & 100,0 \\
\hline 13 años y más & $\ldots$ & 127,9 & 148,9 & 170,7 & $\ldots$ & 125,8 & 164,2 & 214,9 \\
\hline
\end{tabular}

Fuente: CEPAL, sobre la base de tabulaciones especiales de las encuestas de hogares de los países.

CUADRO 9

América Latina (16 países): ingreso laboral de las mujeres jóvenes respecto del ingreso medio de los hombres jóvenes correspondientes, por grupo etarios y nivel educativo, entre alrededor de 1990 y alrededor de 2002 (En promedios simples)

\begin{tabular}{|c|c|c|c|c|c|c|}
\hline \multirow[b]{2}{*}{ Años de estudio } & \multicolumn{3}{|c|}{ Alrededor de 1990} & \multicolumn{3}{|c|}{ Alrededor del 2002} \\
\hline & $\begin{array}{c}\text { 15-19 } \\
\text { años }\end{array}$ & $\begin{array}{c}20-24 \\
\text { años }\end{array}$ & $\begin{array}{c}25-29 \\
\text { años }\end{array}$ & $\begin{array}{c}\text { 15-19 } \\
\text { años }\end{array}$ & $\begin{array}{c}20-24 \\
\text { años }\end{array}$ & $\begin{array}{c}25-29 \\
\text { años }\end{array}$ \\
\hline Total & 91,7 & 81,9 & 76,0 & 87,2 & 80,6 & 76,2 \\
\hline 0-3 años & 94,0 & 76,3 & 62,8 & 75,0 & 62,1 & 56,6 \\
\hline 4-6 años & 79,1 & 64,8 & 62,4 & 83,1 & 67,7 & 57,8 \\
\hline 7-9 años & 83,2 & 68,8 & 61,1 & 83,2 & 68,9 & 58,5 \\
\hline 10-12 años & 104,1 & 85,0 & 71,7 & 89,4 & 78,2 & 69,8 \\
\hline 13 años y más & $\ldots$ & 77,0 & 75,4 & $\ldots$ & 84,4 & 76,4 \\
\hline
\end{tabular}

Fuente: Comisión Económica para América Latina y el Caribe (CEPAL), sobre la base de tabulaciones especiales de las encuestas de hogares de los países.

las mujeres alcanza, en el 2002, al $87 \%$ del ingreso medio en el grupo de 15 a 19 años, al $81 \%$ en el grupo de 20 a 24 años y al $76 \%$ en el grupo de 25 a 29 años (cuadro 9). Por lo tanto, nuevamente se observa que la mayor experiencia potencial, en el caso de las mujeres, no se premia en la misma magnitud que en el caso de los hombres.

Un resultado interesante es que, mientras en muchos estudios — por ejemplo, CEPAL (2001) — se muestra que la brecha de ingresos entre hombres y mujeres, en general, es mayor en los niveles educativos altos que en los bajos e intermedios, esto no ocurre entre los jóvenes. En efecto, la brecha de ingresos de las jóvenes con más alto nivel de educación con respecto a los otros grupos educativos es la más baja en los tres subgrupos etarios juveniles. Esto podría significar que los ingresos relativos de este grupo de mujeres sufren el mayor retroceso posterior, cuando los hombres de alto nivel educativo obtienen elevados premios por su experiencia, típicamente relacionados con promociones, mientras que los premios a la experiencia de las mujeres, debido a las interrupciones de su carrera (por maternidad) y la discriminación, crecerían en menor magnitud. Se puede plantear también, alternativa o complementariamente, la hipótesis de que existe una tendencia a menor discriminación para las mujeres jóvenes más educadas, que crecientemente lograrían defender sus derechos a un pago igual al de los hombres de similar capacidad. ${ }^{23}$

\footnotetext{
${ }^{23}$ Schkolnik (2005, p. 37) muestra pautas muy similares de inserción ocupacional para hombres y mujeres jóvenes de alto nivel educativo.
} 
Esta última hipótesis se vería confirmada por el hecho de que las jóvenes de mejor nivel educativo, de 20 a 29 años, pudieron reducir la brecha de ingreso respecto de sus pares masculinos, mientras la pauta predominante entre los otros grupos educativos fue, por el contrario, una ampliación de las brechas.

Concluyendo este breve repaso de la información estadística más relevante, cabe señalar que, durante el período reciente, la situación laboral de los jóvenes latinoamericanos se ha deteriorado otra vez. Esto obedeció a tendencias generales en los mercados de trabajo de la región, los que en medio de una fuerte volatilidad macroeconómica sufrieron un nuevo empeoramiento de las condiciones de empleo e ingresos. Contrariamente a lo que hubiese podido esperarse sobre la base de las hipótesis de las ventajas competitivas tecnológicas y organizativas de los jóvenes, no se observó una mejoría de su situación laboral con respecto a los adultos. Esto no significa que las expectativas eran completamente erróneas, ${ }^{24}$ pero sí que no corresponden al conjunto de jóvenes sino a grupos específicos.

\section{IV}

\section{Tensiones en la inserción laboral de los jóvenes}

La brecha entre las expectativas de mejoramiento de la inserción laboral de los jóvenes - a causa de aspectos educativos, demográficos, tecnológicos y económicos- y la evolución reciente de esta inserción (véase la sección II anterior) es el marco para una serie de tensiones, muchas de ellas vinculadas entre sí. ${ }^{25}$ Estas tensiones generalmente surgen entre la subjetividad de los jóvenes y la realidad del mercado de trabajo. Dada la gran heterogeneidad de las cohortes juveniles, estas tensiones obviamente no afectan a todos los jóvenes en el mismo grado. Sin embargo, es posible advertir que muchos de ellos las experimentan en todos los países.

Primera tensión: los jóvenes tienen hoy niveles más altos de educación formal que las cohortes etarias anteriores, pero también enfrentan mayores problemas de acceso al empleo. ${ }^{26}$ La causa de esta tensión radica principalmente en la debilidad de la demanda agregada, que ha incidido en que gran parte del nuevo empleo haya surgido en sectores de baja productividad, que se expan-

\footnotetext{
${ }^{24}$ Véase Campusano (2006, p. 97) sobre las características atribuidas a los jóvenes desde la perspectiva empresarial.

${ }^{25}$ La sección IV se basa principalmente en el resultado de una serie de grupos focales - llevados a cabo en el marco del proyecto CEPAL/GTZ, mencionado en la nota al pie 2 - integrados por jóvenes y adultos jóvenes de diferentes niveles educativos. Véase al respecto Espinosa (2006), Sepúlveda (2006) y Palau, Caputo y Segovia (2006), así como los capítulos correspondientes en Carranza (2006) y Chacaltana (2006a).

${ }^{26}$ Se trata de la primera de las tensiones o paradojas analizadas por Martín Hopenhayn en relación con la situación de la juventud latinoamericana, y dadas a conocer, entre otros, en CEPAL/OIJ (2004: 17-21). Las tensiones identificadas en este capítulo, que se concentran en aspectos de la inserción laboral de los jóvenes, pueden leerse en forma complementaria de aquellas.
}

dieron por la presión de la oferta laboral. Claramente, el origen de esta tensión no es que las nuevas generaciones sean "demasiado" educadas o que haya un exceso de jóvenes con altos niveles de educación, ya que el aumento de la brecha salarial en favor de los más educados - observado para el período reciente en América Latina (BID, 2003) — refleja que la demanda laboral ha estado más bien sesgada hacia el personal más calificado. Por el contrario, los avances de la cobertura educacional son insuficientes para alcanzar la equidad y el desarrollo de la región - por ejemplo, en comparación con otros países de ingreso medio- y su calidad es inadecuada. Específicamente, los sistemas de educación y formación para el trabajo han sido débiles en abordar los cambios productivos y socioculturales recientes, de manera que no facilitan la transición al mundo laboral.

Segunda tensión: los jóvenes otorgan una alta valoración al trabajo en sí, pero sus experiencias con empleos concretos suelen ser frustrantes. Si bien se aprecia una creciente percepción funcional del trabajo principalmente como fuente de ingreso - que en algunos casos tiene que competir con otras que prometen ganancias mayores y más fáciles-, para muchos jóvenes este sigue siendo la piedra angular en el desarrollo de su identidad personal, a lo que contribuye el logro de nuevas relaciones sociales en el lugar del empleo. Sin embargo, muchas experiencias de trabajo iniciales no cumplen con las expectativas cifradas en ellas, ya que son muchos los jóvenes que informan de ingresos bajos, poca acumulación de conocimientos y habilidades, amenazas de despido, malos tratos, acoso sexual o relaciones personales desagradables; en fin, 
condiciones que no estimulan a aprovechar el potencial que promete el trabajo para el desarrollo individual y social de los jóvenes.

Tercera tensión, vinculada a la anterior: hay fuertes contradicciones entre las expectativas de los jóvenes sobre los beneficios de la inserción en el mercado laboral y la realidad que viven en él. Las expectativas se centran en la mejoría del bienestar material individual y de la familia paterna, la creación de una base para formar un hogar propio, el reconocimiento social, la posibilidad de contribuir al desarrollo de su país y otras. El cambio de roles de género ha reforzado esta tensión, pues cada vez son más las mujeres jóvenes que intentan aprovechar y desarrollar su potencial para alcanzar una mayor autonomía e independizarse de los roles tradicionales estrechamente relacionados con el hogar. Sin embargo, para muchos jóvenes la realidad del mercado de trabajo no satisface estas aspiraciones o lo hace solo parcialmente. Un elemento clave de las frustraciones que esto crea son los bajos ingresos laborales, que se reflejan en los elevados porcentajes de "trabajadores pobres".

Cuarta tensión: en una visión dinámica, las características del mercado laboral se expresan en la contraposición de las necesidades y preferencias de una trayectoria laboral con una estabilidad mínima de empleo e ingresos - sobre todo a partir de la aspiración de los jóvenes de formar una familia propia- y una realidad laboral en la que prevalece una alta inestabilidad y precariedad. Los jóvenes actuales han hecho sus primeras experiencias laborales en esta "nueva normalidad laboral" (Sepúlveda, 2006) y para algunos ella representa un marco adecuado para sus aspiraciones de autonomía y creatividad. Sin embargo, para la mayoría el mercado laboral no es uno que ofrezca oportunidades múltiples y dinámicas, sino uno que obstaculiza las trayectorias ascendentes y las relaciones laborales estables, lo que unido al debilitamiento de los sistemas de protección social en muchos países, genera una profunda incertidumbre que afecta al desarrollo de la personalidad de los jóvenes y su inclusión social.

Quinta tensión: el cumplimiento de las aspiraciones relacionadas con el mercado de trabajo requiere generalmente un plazo largo, en particular para alcanzar altos niveles educativos. Sin embargo, muchos jóvenes enfrentan urgencias de corto plazo que los empujan a desertar tempranamente del sistema escolar, les impiden retomar sus estudios y los obligan a aceptar cualquier empleo disponible para generar ingresos laborales indispensables para su hogar. Mientras que para los jóvenes provenientes de hogares pobres esta tensión se hace patente a temprana edad, para otros el choque entre las aspiraciones de largo plazo y las urgencias de corto plazo surge con las responsabilidades que conlleva una familia propia. En estos casos, la tensión entre las aspiraciones y la realidad laboral a menudo se "resuelve" traspasando aquellas a la generación siguiente, de manera que los sacrificios del corto plazo deben facilitar que estas aspiraciones se cumplan para los hijos.

Sexta tensión: las mujeres jóvenes están mostrando un interés creciente por alcanzar su propia autonomía, para lo cual el empleo desempeña un papel clave, pero se enfrentan a problemas especiales de inserción laboral. Aunque esta situación es especialmente grave respecto de las mujeres con bajos niveles educacionales, para las que existen muy pocas oportunidades de empleo productivo, las jóvenes presentan indicadores laborales desfavorables en comparación con los jóvenes de similares grupos de edad en todos los niveles educativos. En consecuencia, y a pesar de sus niveles más altos de educación formal, también en el promedio del conjunto de las mujeres jóvenes los indicadores de inserción laboral son inferiores a los de los hombres (CEPAL, 2004, pp. 167-171).

Séptima tensión: la creciente importancia de la combinación del trabajo con los estudios puede generar tensiones negativas, al afectar el rendimiento en ambos campos, o positivas, al abrir el acceso a oportunidades de otro modo negadas. De todas maneras, la importancia cada vez mayor del "aprendizaje continuo" puede implicar una tensión duradera a lo largo de la vida laboral, con efectos potencialmente negativos para el tiempo de libre disposición y la vida familiar y social, especialmente en los países latinoamericanos, con sus largas jornadas laborales.

Octava tensión: los jóvenes viven la tensión entre un discurso "meritocrático" — al que responden con la disposición de hacer grandes esfuerzos y sacrificios personales para avanzar en su educación e inserción laboral- y una realidad del mercado de trabajo en que los contactos personales y las recomendaciones desempeñan con frecuencia un importante papel en el acceso a empleos atractivos. La exclusión laboral de quienes no cuentan con este tipo de capital social refleja una marcada segmentación intrageneracional, que se está profundizando en muchos países debido a crecientes diferencias en la calidad de la educación a la que tienen acceso jóvenes de diferente estatus socioeconómico.

Novena tensión: el mercado exige —entre otros requisitos- experiencia laboral; sin embargo, muchos jóvenes que buscan trabajo por primera vez se encuentran con que, por una parte, les es sumamente difícil acumular experiencia y, por otra, el mercado no reconoce la experiencia adquirida en ocupaciones a las que pueden 
acceder los jóvenes de bajo nivel educativo. A este grupo, por lo tanto, le resulta casi imposible emprender una trayectoria laboral ascendente.

Décima tensión: los jóvenes muestran un interés cada vez mayor en la independencia laboral y el emprendimiento y en un discurso que estimula esta orientación, planteándola —entre otras - como una alternativa de trabajo frente a una baja generación de empleo asalariado. Sin embargo, existen considerables obstáculos para iniciar actividades empresariales (experiencia, crédito y otros), además de un alto riesgo de fracaso, ${ }^{27}$ en circunstancias de que, salvo pocas excepciones, no se han creado aún las instituciones necesarias para apoyar a los jóvenes en emprendimientos de este tipo y mucho menos en el caso de un fracaso. Asimismo, las situaciones de crisis o de bajo crecimiento económico, que limitan la generación de empleo asalariado, restringen también las oportunidades de creación y expansión de nuevas empresas.

Undécima tensión: los jóvenes enfrentan la tensión entre sus preferencias culturales y las pautas exigidas por un mercado de trabajo marcado por la cultura dominante. Ellos perciben procesos de exclusión a causa de su edad y sus expresiones culturales, mientras que el mercado valora la experiencia laboral más de lo que a los jóvenes les parece justo, y no acepta ciertas expresiones subculturales que podrían afectar la imagen de las empresas ante sus clientes y, por ende, sus resultados económicos.

Las tensiones señaladas inciden en los procesos de integración laboral y social. Muchas de ellas se relacionan con una contradicción más profunda entre los sueños y las aspiraciones individuales y colectivas y una realidad social y económica que no facilita su cumplimiento. Esta contraposición puede generar conflictos que se expresarían, con mayor o menor fuerza, tanto a nivel individual como bajo la forma de conflictos sociales con componentes generacionales. Como es obvio, se trata de una tensión dinámica, y los sueños y aspiraciones de una cohorte de jóvenes se desarrollan en direcciones generalmente imprevisibles, pero no desconectadas por completo de la realidad cambiante de las sociedades. Lo mismo sucede si se comparan las sucesivas cohortes juveniles actuales con las que están por venir, cada una de las cuales suele desarrollar diferencias y modificaciones de los valores y objetivos característicos de su antecesora etaria.

\section{V \\ Desafíos para mejorar la inserción laboral de los jóvenes}

Para avanzar significativamente en la inserción laboral de los jóvenes y enfrentar las tensiones antes identificadas es indispensable contar con un entorno macroeconómico propicio. Ningún programa que mejore la empleabilidad de los jóvenes (es decir, el desarrollo de conocimientos, habilidades y competencias para el empleo), aumente la eficiencia de la intermediación laboral o intervenga en otros aspectos de la inserción en el trabajo puede tener resultados satisfactorios sin una demanda laboral dinámica, producto de tasas de crecimiento económico altas y estables y de expectativas que induzcan a las empresas a contratar más personal, y sin un contexto promisorio para la creación de trabajos independientes con perspectivas de trayectorias en subida.

${ }^{27}$ Véase más adelante el apartado 3 de la sección V.
Muchas de las tensiones descritas tienen que ver con un mercado de trabajo volátil y precario para muchas personas que buscan empleo. Las antiguas previsiones y mecanismos de protección — de por sí accesibles solo a una parte de la fuerza de trabajo de la región- han perdido gran parte de su capacidad regulatoria. En consecuencia, otro reto pendiente es el de establecer una nueva regulación del mercado ocupacional, que fomente relaciones laborales en las que se promuevan, entre otros, mecanismos de protección acordes con las nuevas realidades económicas. ${ }^{28}$

Sin embargo, no todas las tensiones observadas en los procesos de inserción laboral se relacionan con la debilidad del crecimiento o con la institucionalidad del

\footnotetext{
${ }^{28} \mathrm{Al}$ respecto, véase en CEPAL (2007) el análisis de un pacto de cohesión social que abarca intervenciones en el mercado de trabajo, en este sentido.
} 
mercado de trabajo. Aun más, no es posible imaginar "soluciones" que pongan fin a todas ellas de manera satisfactoria para todos los involucrados. Además, algunas se relacionan estrechamente con conflictos intergeneracionales dinámicos, que difícilmente pueden ser objeto de intervención de políticas públicas.

A continuación, se examinan algunas opciones de políticas para fomentar la inserción laboral de los jóvenes. Este examen se organiza en torno a los cuatro ejes (conocidos en inglés como "the $4 E$ ") que propuso el Grupo de Alto Nivel de la Red de Empleo para los Jóvenes y que son los siguientes: empleabilidad, equidad de género, espíritu empresarial y generación de empleo (Naciones Unidas, 2001). ${ }^{29}$

Cabe recordar que cualquier intervención encaminada a mejorar la inserción laboral de los jóvenes debe considerar la gran heterogeneidad de la juventud de la región, que se expresó claramente en los datos presentados en la sección III. No existe un problema de inserción laboral común para todos los jóvenes, sino una variedad de problemas específicos. Los retos que enfrentan los jóvenes de diferente género y características educativas, socioeconómicas, culturales, étnicas y otras, difieren ostensiblemente, de manera que es preciso encontrar respuestas adecuadas para diferentes necesidades específicas. Además, se necesita una mejor coordinación de los actores públicos, privados y no gubernamentales, a nivel nacional y local, que relacione los esfuerzos de los jóvenes y sus familias con un entorno más favorable para su inserción laboral.

\section{Empleabilidad}

Este apartado gira en torno a los conceptos de capital humano, capital social y capital cultural. Mientras los economistas tradicionalmente trabajan con el concepto de capital humano y recientemente han "descubierto" el capital social, los sociólogos suelen distinguir entre el capital social y el capital cultural, el segundo de los cuales incluye los aspectos de educación, capacitación y experiencia laboral que los economistas definen como determinantes del capital humano. Aquí, en cambio, se entiende que para lograr una inserción laboral exitosa se requiere capital humano (educación y capacitación de buena calidad), capital social (relaciones sociales basadas en la confianza, la cooperación y la reciprocidad)

\footnotetext{
${ }^{29}$ En las deliberaciones de la Cumbre de Empleo Juvenil —Youth Employment Summit (YES) — se propuso añadir tres ejes más: sostenibilidad ambiental, empoderamiento y educación.
}

y capital cultural (manejo de los códigos establecidos por la cultura dominante).

\section{a) Capital humano}

Una buena educación y formación para el trabajo son elementos clave en el desarrollo del capital humano y la empleabilidad de los jóvenes. Sin embargo, en los sistemas educativos de la región persisten múltiples deficiencias de cobertura y calidad. ${ }^{30}$

Los países de América Latina han puesto un marcado acento en universalizar la educación primaria, llegando en el 2001 a una cobertura del 93\% (Naciones Unidas, 2005, p. 90). No obstante, persisten insuficiencias en la cobertura de la educación secundaria —que en el 2001 alcanzó un promedio simple de $65 \%$ entre los países de la región (Naciones Unidas, 2005, p. 109)—y de la preescolar, siendo esta última fundamental para compensar las desventajas de los niños de hogares pobres.

Además, los sistemas educativos de la región padecen reconocidamente de problemas de niveles y brechas de calidad (Labarca, 2004; Naciones Unidas, 2005), lo que se ha manifestado, entre otras cosas, en una menor valoración de las credenciales académicas. Existe por lo tanto una presión "de arriba hacia abajo": jóvenes con un título no consiguen trabajo en ocupaciones para las que están capacitados, por lo que ocupan un espacio laboral inferior, desplazando a los jóvenes que se han preparado para estas ocupaciones (Novick, 2004). Además, los jóvenes enfrentan la segmentación del sistema educativo, que favorece a los graduados de colegios y universidades reconocidos por la calidad de su enseñanza pero cuyo acceso está restringido por obstáculos financieros (CEPAL/OIJ, 2004).

Los jóvenes han comprendido claramente lo importante que es la educación, pero con bastante frecuencia opinan que ni la educación ni la formación profesional los prepara adecuadamente para el ámbito laboral, por la falta de vínculos entre el currículo escolar y el mundo del trabajo. ${ }^{31}$ Además, como ya hemos señalado, con frecuencia las urgencias de corto plazo obligan a los jóvenes a insertarse tempranamente en el mercado de trabajo, lo que se refleja, por ejemplo, en el aumento del porcentaje de jóvenes que estudian y trabajan o que

\footnotetext{
${ }^{30}$ Abdala (2004, p. 31) menciona las siguientes deficiencias del sistema educativo de la región: ingreso tardío a la enseñanza primaria $(20 \%$ de los jóvenes); altas tasas de repetición (40\% en el primer año); atraso escolar (50\% en algún momento del ciclo), y ausentismo en la enseñanza secundaria (ingreso del 50\% de los habilitados). Véase también CEPAL/OIJ (2004).

${ }^{31}$ Romero-Abreu Kaup y Weller (2006) examinan algunas ideas al respecto.
} 
estudian y buscan trabajo. Cuando el trabajo afecta el rendimiento escolar, debido a jornadas laborales extensas o extenuantes que restringen la capacidad de aprendizaje, este aumento representa una tendencia adversa que hipoteca el futuro de los jóvenes.

Por otra parte, si los jóvenes asisten al sistema escolar y a la vez trabajan en formas que no afecten significativamente su rendimiento en los estudios -durante las vacaciones o con un horario de trabajo acotado-, no solo generarán ingresos, sino que se acercarán al mundo laboral, ya que adquirirán y pondrán en práctica destrezas que no son gravitantes en el ámbito escolar (Krauskopf, 2003). Además, puesto que las transiciones al mundo adulto y laboral suelen ser largas (Sepúlveda, 2006), una combinación adecuada de estudio y trabajo puede ayudar a los jóvenes a desarrollar estrategias laborales individuales y generar la autoestima y el reconocimiento de las propias capacidades que les son necesarios para dar los primeros pasos en la actividad laboral.

Es de suponer que la combinación de trabajo y estudios más benigna es más usual entre jóvenes de hogares de ingresos más altos, mientras que los jóvenes procedentes de hogares pobres enfrentan la modalidad adversa. En los casos de jóvenes de escasos recursos, los programas de transferencias condicionadas - que reducen la necesidad del trabajo infantil y juvenil— son un instrumento adecuado para limitar el impacto negativo de las urgencias de corto plazo. Además, habría que mejorar la flexibilidad del sistema educativo y de la legislación laboral (contratos de jornada parcial con los beneficios sociales correspondientes, posibilidad de manejo flexible del tiempo de trabajo), a fin de permitir la combinación de trabajo y estudios bajo condiciones benignas.

\section{b) Capital social}

Se ha puesto de relieve que el acceso al capital social a la vez contribuye a causar (por su distribución desigual) y resolver (al mejorar su acumulación por grupos desaventajados) los problemas de inequidad de las sociedades latinoamericanas (Durston, 2003).

Con respecto al acceso al mercado de trabajo, llama la atención la importancia que tanto los empresarios como los jóvenes atribuyen a las recomendaciones de terceros para la contratación. ${ }^{32}$ En un mercado laboral al que le falta transparencia, algo bastante generalizado

\footnotetext{
32 Véase al respecto los resúmenes que aparecen en Espinosa (2006, p. 35) y Campusano (2006, p. 94).
}

en América Latina, este comportamiento puede resultar una solución subóptima para las empresas, especialmente aquellas de menor tamaño que quieren evitar costosos procesos de selección.

Sin embargo, estas prácticas implican una dinámica de exclusión para aquellos jóvenes que carecen de los contactos necesarios. ${ }^{33}$ En estas circunstancias, la distribución desigual de capital social predetermina poderosamente las oportunidades de acceso a empleos productivos y, por lo tanto, las futuras trayectorias laborales de muchos jóvenes. Esto genera grandes frustraciones, ya que en la realidad del mercado de trabajo se menosprecian los esfuerzos de educación y capacitación de quienes no cuentan con los contactos sociales requeridos. Además, los que consiguen su inserción laboral de esta manera refuerzan aún más su capital social en detrimento de los jóvenes que carecen de esos contactos iniciales.

Con el fin de aumentar la eficiencia de la intermediación laboral y otorgar más equidad a los procesos de inserción laboral, es necesario mejorar la transparencia del mercado de trabajo, por ejemplo, mediante la certificación de competencias, agencias de (pre)selección de personal, apoyo al desarrollo de estrategias laborales de jóvenes en situación de desventaja, y mejoramiento cuantitativo y cualitativo de los sistemas de intermediación, aprovechando las nuevas tecnologías de información y comunicación.

\section{c) Capital cultural}

Para las empresas, las actitudes y valores de los postulantes desempeñan un papel clave en la contratación de sus trabajadores, y en muchos de estos aspectos los jóvenes son habitualmente mal evaluados (Campusano, 2006). En efecto, un importante obstáculo para la inserción laboral de muchos jóvenes es su falta de manejo de los códigos culturales vigentes y requeridos en el mundo laboral. A este respecto, habría que diferenciar entre los problemas causados por desconocimiento y los conflictos que surgen de tensiones subculturales. Con relación a los primeros, claramente muchos jóvenes no conocen las actitudes, modalidades y formas de presentación valoradas por las empresas, lo que se expresa, por ejemplo, en una mala presentación de su currículum vítae y en fallidas

\footnotetext{
${ }^{33}$ En los grupos focales con jóvenes y adultos jóvenes, el tema de la contratación privilegiada surgió con frecuencia. En los países sin una carrera de servicio civil independiente de las fuerzas políticas de turno, se destacó la importancia de los contactos políticos para conseguir un empleo en el sector público (véase, por ejemplo, Palau, Caputo y Segovia, 2006).
} 
entrevistas de contratación. Indudablemente, la capacitación en esta área puede ser una inversión eficiente para mejorar las perspectivas de inserción laboral de jóvenes que poseen las calificaciones requeridas para ocupar un determinado puesto.

Tanto en los estudios sobre los jóvenes como en los de las empresas se subraya una actitud juvenil proactiva bastante generalizada. De hecho, muchos jóvenes destacan la relevancia del esfuerzo personal - a pesar de muchos otros factores adversos (Sepúlveda, 2006). Esta actitud es corroborada por las empresas que dan cuenta de la buena disposición de los jóvenes frente al trabajo en general y a nuevos retos, en particular, entre las características positivas de las nuevas generaciones (Campusano, 2006, p. 98).

Sin embargo, hay muchos jóvenes que perciben que son víctimas de discriminación a causa de sus expresiones culturales (ropa, corte de pelo, adornos corporales), las que suelen ser rechazadas por el mundo del trabajo, sobre todo en el sector formal. ${ }^{34}$ Como es obvio, se trata de una tensión permanente, aunque en paralelo se dan procesos de filtración de expresiones culturales desde las subculturas hacia la cultura dominante. Es posible que en algún momento la cultura dominante llegue a tolerar las expresiones antes excluidas y castigadas, e incluso a incorporarlas; a su vez, cada generación crea sus propias expresiones culturales que causan nuevas tensiones con el entorno dominante. Cada joven deberá decidir hasta dónde y en qué momento está dispuesto a transar con las exigencias del mundo laboral tradicional, o si se esfuerza por encontrar un modo creativo propio, concordante con sus creencias y valores, que le permita obtener ingresos. ${ }^{35}$

De todas maneras, recientemente algunos cambios en la estructura productiva y laboral, como el surgimiento y la expansión de múltiples actividades en el sector de los servicios, el trabajo a tiempo parcial y estructuras laborales menos jerárquicas, ofrecen a ciertos jóvenes nuevas oportunidades más compatibles con sus intereses y preferencias, entre ellas, el trabajo en equipo y la flexibilidad horaria.

\footnotetext{
${ }^{34}$ Véanse las opiniones de empresarios salvadoreños citadas en Vega y Carranza (2006).

35 Hay que tener en cuenta que la tolerancia hacia ciertas expresiones culturales no puede ser unilateral. Además, habría que distinguir entre expresiones de una ideología intolerante y represiva, y la existencia de diferentes "escenarios" sociales que manejan códigos diferentes, sin que esto necesariamente implique una discriminación.
}

\section{Equidad de género}

Existen barreras específicas que impiden el acceso de muchas mujeres al mercado laboral, sobre todo debido a obstáculos relacionados con pautas tradicionales de la división de trabajo: las mujeres son relegadas al ámbito privado y están a cargo de la reproducción, mientras los hombres actúan en el ámbito público y son responsables del trabajo productivo. Destacan aquí, por una parte, los roles asignados a las jóvenes de familias de escasos recursos que viven en zonas rurales, en muchos casos encargadas del cuidado de los hermanos menores u otros oficios del hogar. Y por otra, las madres jóvenes con pocos recursos de zonas urbanas, que suelen tener dificultades para encontrar y financiar servicios de atención a sus hijos durante la jornada de trabajo. Con el objetivo de garantizar la igualdad de oportunidades de género a la hora de la integración en el mundo laboral, las políticas de fomento de la inserción laboral juvenil deben considerar estas necesidades específicas de los distintos grupos de mujeres jóvenes. Cabe resaltar que la integración de los oficios del hogar y la inserción laboral no puede interpretarse como tarea exclusivamente de las mujeres (Batthyány, 2004).

Por otra parte, hay que tomar en cuenta la perspectiva de género en la formación profesional, tanto para mejorar la calidad de los programas en ocupaciones a las que las mujeres han tenido acceso tradicionalmente como para ampliar su acceso a las demás (Fawcett y Howden, 1998).

Otra medida para mejorar la empleabilidad de las mujeres jóvenes es el apoyo a la definición de estrategias laborales personales. Estas estrategias abarcan, entre otros componentes, el establecimiento temprano de contactos en el mundo laboral y su conocimiento, así como la mejoría de la información sobre el mercado de trabajo en general y sobre las opciones laborales y de capacitación. Con ello contribuyen al desarrollo del capital cultural y social, y de la autoestima, todos elementos muy importantes para la inserción laboral, en los cuales las mujeres jóvenes, sobre todo de hogares de bajos ingresos, suelen tener marcadas desventajas.

En general, las políticas de empleo, específicamente las dirigidas a los jóvenes, deben adoptar un enfoque transversal de género, reconociendo las múltiplas trabas y desigualdades que enfrentan las mujeres en el acceso al mercado de trabajo y en el mundo laboral mismo (Abramo, 2006). En parte estos problemas se deben a actitudes discriminatorias, que deben identificarse y enfrentarse. En este sentido, las leyes antidiscriminatorias tienen tanta relevancia (o 


\begin{tabular}{|c|c|c|}
\hline & Jóvenes & Adultos \\
\hline \multicolumn{3}{|c|}{$\begin{array}{l}\text { Chile, cambio entre inicio y fin de periodo de } 18 \text { meses, 1996-2003 } \\
\text { Independientes }\end{array}$} \\
\hline Total (inicio del período) & 12,5 & 27,8 \\
\hline Sin cambio & 7,5 & 22,7 \\
\hline Cambio (asalariados u otros) & 5,0 & 5,1 \\
\hline \multicolumn{3}{|c|}{$\begin{array}{l}\text { Ecuador, cambios entre abril-mayo de } 2000 \text { y abril-mayo de } 2002 \\
\text { Independientes }\end{array}$} \\
\hline Total (inicio del período) & 10,1 & 37,5 \\
\hline Sin cambio & 6,6 & 31,2 \\
\hline Cambio (asalariados u otros) & 3,5 & 6,3 \\
\hline \multicolumn{3}{|l|}{$\begin{array}{l}\text { Perú, cambios entre } 1998 \text { y } 2001 \\
\quad \text { Independientes }\end{array}$} \\
\hline Total (inicio del período) & 40,8 & 63,0 \\
\hline Sin cambio & 17,8 & 44,3 \\
\hline Cambio (asalariados u otros) & 23,0 & 18,7 \\
\hline
\end{tabular}

Fuente: Romero-Kaup Abreu y Weller (2006, p. 263).

más) para estimular los procesos de cambio social y cultural hacia sociedades más equitativas como para corregir transgresiones puntuales, especialmente porque la discriminación suele disfrazarse con argumentos racionales de eficiencia. ${ }^{36}$

\section{Espíritu emprendedor}

Las estructuras productivas están en proceso de rápida transformación, a lo que contribuyen en gran medida las nuevas tecnologías de información y comunicación. Estas tecnologías no solo generan y exigen una mayor flexibilidad en los mercados — entre ellos, el laboral-, sino que también facilitan la inserción productiva independiente con inversiones de capital mucho menores que en la pauta productiva anterior. Estas oportunidades tecnoproductivas se ven reflejadas en el creciente interés de muchos jóvenes por tener una mayor independencia laboral, en un entorno en el que el empleo asalariado se caracteriza por una mayor precariedad e inestabilidad (Espinosa, 2006). En parte recogiendo estas tendencias, recientemente se ha visto en la promoción de las microempresas y el empleo por cuenta propia una estrategia para abordar el problema del desempleo juvenil.

\footnotetext{
${ }^{36}$ Bien se sabe que las leyes y la fiscalización de su cumplimiento no bastan para eliminar la discriminación, pero son instrumentos útiles.
}

Sin embargo, muchas de las microempresas están ligadas a la supervivencia y no son manifestaciones de la vocación emprendedora de los jóvenes (Messina, 2001). Además, hay que tomar en cuenta que las situaciones de escaso dinamismo económico no solo afectan la generación de empleo por parte de las empresas ya existentes, sino que también limitan las oportunidades de establecer y mantener nuevos negocios.

Los datos disponibles muestran que los jóvenes que aspiran a la independencia laboral y la creación de sus propias empresas enfrentan mayores obstáculos que los adultos. En efecto, los ocupados por cuenta propia o como empleadores son una proporción notoriamente menor entre los jóvenes que entre los adultos, lo que no sorprende ya que muchos jóvenes, aunque tengan interés en independizarse, prefieren primero acumular experiencia laboral en un empleo asalariado. Más importante aún es que hay una mayor inestabilidad entre los jóvenes independientes que entre los adultos. Como se puede apreciar sobre la base de los datos presentados en el cuadro 10 para Chile, Ecuador y Perú, una proporción significativamente más alta de jóvenes que trabajaron como independientes - es decir, por cuenta propia o como empleadores - cambian de categoría, en comparación con lo que se observa para los adultos. Por ejemplo, en Chile, a lo largo de un período de 18 meses, el 40\% de los jóvenes que trabajaron como independientes al inicio del período, hacia el fin de ese lapso se cambiaron 
a alguna categoría dependiente, mientras que entre los adultos la proporción solo alcanzó al 18\%. En Ecuador, en tanto, para un período de dos años, los porcentajes son de $35 \%$ y $17 \%$, respectivamente; y en Perú, para un período de tres años, de $56 \%$ y $30 \%$, respectivamente. En resumen, la proporción de los jóvenes independientes que dejan de serlo en los tres países duplica el porcentaje de los adultos. ${ }^{37}$

Estos datos muestran lo riesgosa que sería una inversión masiva en programas de fomento de microempresas juveniles. Definitivamente, no se trata de una solución universal para mejorar la inserción laboral de los jóvenes. Por otra parte, es aconsejable reorientar la educación hacia el fomento del espíritu emprendedor que no se propone exclusivamente la meta de crear empresarios, sino también la de estimular habilidades, competencias y actitudes útiles para los jóvenes en el mundo laboral en general, la vida del barrio, el trabajo voluntario y las organizaciones políticas, sociales, culturales, deportivas u otras.

En algunos de los jóvenes, estas actividades estimularán el interés por aventurarse en el mundo empresarial. Las políticas y programas de apoyo a estos empresarios potenciales deben ser diferenciados según el grupo de jóvenes al que vayan dirigidos y deben vincularse con las políticas generales de fomento a la creación de empresas.

\section{Creación de empleo}

Si bien en algunas empresas se afirma que contratan a jóvenes con una buena formación, aunque no tengan experiencia, de hecho para la inserción laboral de los jóvenes se suele plantear exigencias adicionales (de experiencia, conexiones personales, manejo de pautas culturales, esfuerzo individual y otras). Entonces, más allá de las correlaciones positivas a nivel agregado, muchos jóvenes perciben que la relación entre escolaridad y acceso a empleo productivo se está debilitando. Así, a los jóvenes que buscan trabajo por primera vez se les exige educación y experiencia para ingresar al mercado laboral, pero ante la falta de experiencia, no se les da la oportunidad de adquirirla. Por lo demás, las deficiencias en el funcionamiento del mercado laboral - falta de transparencia, mecanismos de exclusión y

\footnotetext{
${ }^{37}$ En la comparación entre el inicio y el fin del período correspondiente, no se informa sobre lo acontecido en el intermedio, ni tampoco si una persona que ha mantenido su categoría de ocupación se encuentra trabajando en la misma actividad. Sin embargo, en los datos se observan tendencias generales significativas.
}

discriminación - agudizan las dificultades para que determinados grupos accedan a ese mercado. De ahí la importancia de fomentar el acceso a un primer empleo con perspectivas de una trayectoria laboral ascendente. En los últimos años han sido varios los países que han creado programas para estimular con diferentes instrumentos un primer empleo formal. ${ }^{38}$

De hecho, dado el aumento de las relaciones laborales inestables, la inserción inicial en el mundo del trabajo no garantiza necesariamente una trayectoria laboral ascendente; pero un primer empleo formal puede dar señales potentes al mercado respecto a la acumulación de experiencias y habilidades útiles. Entre ellas destacan el aprendizaje de nuevas destrezas, generalmente por la práctica más que por esquemas formales de capacitación; la acumulación de experiencia laboral y capital cultural, y la posibilidad de relacionarse con otras personas, jóvenes y adultas, en un nuevo contexto de socialización y de establecer así redes sociales que trasciendan el entorno familiar.

Por otra parte, en varios países se han introducido contratos especiales, con derechos laborales reducidos (en materia de salario, cobertura social y condiciones de despido), para fomentar la contratación de algunos grupos, sobre todo jóvenes. En este último caso, es imperativo que tales contratos incluyan medidas de capacitación verificables, de manera que no se sustituya simplemente mano de obra adulta por mano de obra joven peor remunerada. ${ }^{39}$ Muchos jóvenes estiman que estas modalidades de contratación, así como las pasantías, son más un mecanismo para aprovecharse de su fuerza de trabajo a bajo costo que un medio para adquirir conocimientos y habilidades relevantes para su futura vida laboral. De hecho, la evidencia indica que muchas empresas no cumplen con los requisitos establecidos en la legislación respecto de las actividades de capacitación (Chacaltana, 2006a). Dado que este tipo de incentivos salariales y no salariales - como también los fiscales, en el caso de subsidios a la contratación - solamente tienen justificación social si generan una inversión en capital humano, la fiscalización del cumplimiento de las obligaciones de los involucrados es sumamente importante. Esto es más factible en esquemas duales de formación profesional, donde se establece una interacción entre el aprendizaje teórico y el práctico. ${ }^{40}$

\footnotetext{
${ }^{38}$ En 2007, México ha realizado el esfuerzo más reciente para estimular el "primer empleo".

${ }^{39}$ Lo mismo vale para salarios mínimos diferenciados por edad.

${ }^{40}$ Por otra parte, no se aconseja la incorporación de jóvenes en los programas de empleo de emergencia, debido a que ahí generalmente
} 
En conclusión, para mejorar la inserción laboral de los jóvenes es necesario establecer un círculo virtuoso entre un contexto más favorable — donde destacan las condiciones macroeconómicas que estimulan el crecimiento económico y por ende la demanda laboral, una nueva institucionalidad del mercado de trabajo y un reforzamiento del capital humano, social y cultural de los jóvenes-, sobre todo de aquellos en situación de desventaja. Las medidas para mejorar las condiciones de la inserción laboral de las mujeres jóvenes, estimular el espíritu emprendedor y crear empleo harían una importante contribución complementaria. Así se generarían y aprovecharían más oportunidades de iniciar trayectorias laborales ascendentes y de reducir las profundas desigualdades que caracterizan a la región.

\section{Bibliografía}

Abdala, E. (2004): Formación y empleabilidad de jóvenes en América Latina, en M. Molpeceres Pastor (coord.), Identidades y formación para el trabajo, Montevideo, Organización Internacional del Trabajo (OIT)/Centro Interamericano de Investigación y Documentación sobre Formación Profesional (CINTERFOR).

Abramo, L. (comp.) (2006): Trabajo decente y equidad de género en América Latina, Santiago de Chile, Organización Internacional del Trabajo (OIT).

Banco Mundial (2007): Informe sobre el desarrollo mundial 2007: el desarrollo y la nueva generación, Bogotá, D.C., Mayol Ediciones.

Batthyány, K. (2004): Cuidado infantil y trabajo: ¿un desafio exclusivamente femenino? Una mirada desde el género y la ciudadanía social, Montevideo, Centro Interamericano de Investigación y Documentación sobre Formación Profesional (CINTERFOR).

BID (Banco Interamericano de Desarrollo) (2003): Se buscan buenos empleos, Washington, D.C.

Blanchflower, D.G. y R.B. Freeman (comps.) (2000): Youth Employment and Joblessness in Advanced Countries, Chicago, The University of Chicago Press.

Cacciamali, M.C. (2005): Mercado de trabajo juvenil: Argentina, Brasil y México, $\mathrm{N}^{\circ}$ 2005/02, Ginebra, Organización Internacional del Trabajo (OIT).

Campusano, C.L. (2006): El mercado laboral y los jóvenes: una mirada del empresariado, en J. Weller, Los jóvenes y el empleo en América Latina: desafios y perspectivas ante el nuevo escenario laboral, Bogotá, D.C., Comisión Económica para América Latina y el Caribe (CEPAL)/Mayol ediciones.

Carranza, M. (comp.) (2006): Oferta, demanda e intermediación laboral: aportes para la integración de jóvenes al mercado de trabajo salvadoreño, LC/R.2130, San Salvador, Comisión Económica para América Latina y el Caribe (CEPAL)/Instituto Universitario de Opinión Pública (IUDOP).

CEPAL (Comisión Económica para América Latina y el Caribe) (2001): Panorama social de América Latina, 2000-2001, LC/G.2138-P, Santiago de Chile, octubre. Publicación de las Naciones Unidas, $\mathrm{N}^{\circ}$ de venta: S.01.II.G.141.

(2004): Panorama social de América Latina 2004, LC/L.2220-

$\mathrm{P}$, Santiago de Chile, noviembre. Publicación de las Naciones Unidas, $\mathrm{N}^{\circ}$ de venta: S.04.II.G.148.

(2006): Balance preliminar de las economías de América Latina y el Caribe 2006, LC/G.2327-P, Santiago de Chile, diciembre. Publicación de las Naciones Unidas, $\mathrm{N}^{\mathbf{o}}$ de venta: S.06.II.G.141.

acumulan poca experiencia relevante y la participación en estos programas frecuentemente tiene un efecto estigmatizante en el mercado de trabajo, lo que dificultaría la inserción laboral posterior.
(2007): Cohesión social: inclusión y sentido de pertenencia en América Latina y el Caribe, LC/G.2335/Rev. 1, Santiago de Chile.

CEPAL/OIJ (Comisión Económica para América Latina y el Caribe/ Organización Iberoamericana de Juventud) (2003): Juventud e inclusión social en Iberoamérica, LC/R.2108, Santiago de Chile.

(2004): La juventud en Iberoamérica: tendencias y urgencias, LC/L.2180, M. Hopenhayn (coord.), Santiago de Chile.

Chacaltana, J. (2006a): Empleos para los jóvenes, LC/R.2129, Lima, Centro de Estudios para el Desarrollo y la Participación (CEDEP)/Comisión Económica para América Latina y el Caribe (CEPAL)/Sociedad Alemana de Cooperación Técnica (GTZ).

(2006b): Trayectorias laborales de jóvenes peruanos, en J. Weller, Los jóvenes y el empleo en América Latina: desafíos y perspectivas ante el nuevo escenario laboral, Bogotá, D.C., Comisión Económica para América Latina y el Caribe (CEPAL)/ Mayol ediciones.

Charlín, M. y J. Weller (comps.) (2006): Juventud y mercado laboral: brechas y barreras, LC/R.2131, Santiago de Chile, Comisión Económica para América Latina y el Caribe (CEPAL)/Facultad Latinoamericana de Ciencias Sociales (FLACSO).

Diez de Medina, R. (2001): Jóvenes y empleo en los noventa, Montevideo, Organización Internacional del Trabajo (OIT)/ Centro Interamericano de Investigación y Documentación sobre Formación Profesional (CINTERFOR).

Durston, J. (2003): Capital social: parte del problema, parte de la solución, su papel en la persistencia y en la superación de la pobreza en América Latina y el Caribe, en R. Atria, M. Siles y otros (comps.), Capital social y reducción de la pobreza en América Latina y el Caribe: en busca de un nuevo paradigma, LC/G.2194-P, Comisión Económica para América Latina y el Caribe (CEPAL). Publicación de las Naciones Unidas, $\mathrm{N}^{\mathbf{o}}$ de venta: S.03.II.G.03.

Espinosa, B. (2006): Transformaciones y continuidades en el mundo del trabajo en América Latina desde la perspectiva de los jóvenes: introducción y síntesis de los estudios por país, en J. Weller, Los jóvenes y el empleo en América Latina: desafíos y perspectivas ante el nuevo escenario laboral, Bogotá, D.C., Comisión Económica para América Latina y el Caribe (CEPAL)/Mayol ediciones.

Fajnzylber, E. y G. Reyes (2005): Dinámica del empleo juvenil: resultados preliminares con datos del seguro de cesantía, En foco, $\mathrm{N}^{\circ} 54$, Santiago de Chile, Expansiva.

Fawcett, C. (2002): Los jóvenes latinoamericanos en transición: un análisis sobre el desempleo juvenil en América Latina y el Caribe, serie Documentos de trabajo mercado laboral, Washington, D.C., Departamento de Desarrollo Sostenible, Banco Interamericano de Desarrollo (BID).

Fawcett, C. y S. Howden (1998): El tema de género en los programas de formación técnica y profesional, Women in Development 
(WID), $\mathrm{N}^{\mathrm{o}}$ 103, Washington, D.C., Departamento de Desarrollo Sostenible, Banco Interamericano de Desarrollo (BID).

Henríquez, H. y V. Uribe-Etcheverría (2003): Trayectorias laborales: de la certeza a la incertidumbre, Cuaderno de investigación, No 18, Santiago de Chile, Departamento de Estudios, Dirección del Trabajo.

Hopenhayn, M. (2004): El nuevo mundo del trabajo y los jóvenes, Revista de estudios sobre juventud, año 8, No 20, México, D.F., Jóvenes.

Krauskopf, D. (2003): Proyectos, incertidumbre y futuro en el período juvenil, Archivos argentinos de pediatría, vol. 101, $\mathrm{N}^{\mathrm{o}} 6$.

Labarca, G. (2004): Educación y capacitación para mercados del trabajo cambiantes y para la inserción social, en C. Jacinto (coord.), ¿Educar para qué trabajo? Discutiendo rumbos en América Latina, Buenos Aires, Ediciones La Crujía/Red ETIS.

Martínez Valle, L. (comp.) (2006), Jóvenes y mercado de trabajo en el Ecuador, Santiago de Chile, Facultad Latinoamericana de Ciencias Sociales (FLACSO)/Comisión Económica para América Latina y el Caribe (CEPAL).

Messina, G. (2001): Modelos de formación en las microempresas: en busca de una tipología, en E. Pieck (coord.), Los jóvenes y el trabajo: la educación frente a la exclusión social, México, D.F., Universidad Iberoamericana.

Naciones Unidas (2001): Recomendaciones del Grupo de Alto Nivel de la Red de Empleo de los Jóvenes, Carta dirigida al Presidente de la Asamblea General por el Secretario General, A/56/422, Nueva York, 28 de septiembre.

(2004): Informe sobre la juventud mundial 2003: la situación mundial de los jóvenes, Ginebra.

(2005): Objetivos de desarrollo del milenio. Una mirada desde América Latina y el Caribe, LC/G.2331, J.L. Machinea, A. Bárcena y A. León (coords.), Santiago de Chile, Comisión Económica para América Latina y el Caribe (CEPAL). Publicación de las Naciones Unidas, $\mathrm{N}^{\mathrm{o}}$ de venta: S.05.II.G.107.

Novick, M. (2004): Transformaciones recientes en el mercado de trabajo argentino y nuevas demandas de formación, en C. Jacinto (coord.), ¿Educar para qué trabajo? Discutiendo rumbos en América Latina, Buenos Aires, Red ETIS.

OCDE (Organización de Cooperación y Desarrollo Económicos) (2002): Employment Outlook 2002, París.

O'Higgins, N. (2001): Desempleo juvenil y política de empleo. Una perspectiva global, Ginebra, Organización Internacional del Trabajo (OIT).
OIT (Organización Internacional del Trabajo) (2006): Global Employment Trends for Youth, Ginebra.

Palau, M., L. Caputo y D. Segovia (2006): Paraguay: expectativas y estrategias laborales de los jóvenes, en J. Weller, Los jóvenes y el empleo en América Latina: desafíos y perspectivas ante el nuevo escenario laboral, Bogotá, D.C., Comisión Económica para América Latina y el Caribe (CEPAL)/Mayol ediciones.

Romero-Abreu Kaup, P. y J. Weller (2006): Políticas de fomento de la inserción laboral de los jóvenes, en J. Weller, Los jóvenes y el empleo en América Latina: desafios y perspectivas ante el nuevo escenario laboral, Bogotá, D.C., Comisión Económica para América Latina y el Caribe (CEPAL)/Mayol ediciones.

Schkolnik, M. (2005): Caracterización de la inserción laboral de los jóvenes, serie Políticas sociales, No 104, LC/L.2257-P, Santiago de Chile, Comisión Económica para América Latina y el Caribe (CEPAL), marzo. Publicación de las Naciones Unidas, $\mathrm{N}^{\circ}$ de venta: S.05.II.G.15.

Sepúlveda, L. (2006): Incertidumbre y trayectorias complejas: un estudio sobre expectativas y estrategias laborales de jóvenes y adultos jóvenes en Chile, en J. Weller, Los jóvenes y el empleo en América Latina: desafíos y perspectivas ante el nuevo escenario laboral, Bogotá, D.C., Comisión Económica para América Latina y el Caribe (CEPAL)/Mayol ediciones.

Tokman, V.E. (2003): Desempleo juvenil en el Cono Sur: causas, consecuencias y políticas, Santiago de Chile, Fundación Friedrich Ebert.

Vega, L. y M. Carranza (2006): Experiencia y visión de los empresarios salvadoreños sobre la inserción laboral de los jóvenes, en J. Weller, Los jóvenes y el empleo en América Latina. Desafíos y perspectivas ante el nuevo escenario laboral, Bogotá, D.C., CEPAL/ Mayol ediciones/ GTZ.

Weller, J. (2003): La problemática inserción laboral de los y las jóvenes, serie Macroeconomía del desarrollo, $\mathrm{N}^{\circ}$ 28, LC/L.2029-P, Santiago de Chile, Comisión Económica para América Latina y el Caribe (CEPAL), diciembre. Publicación de las Naciones Unidas, $\mathrm{N}^{\mathrm{o}}$ de venta: S.03.II.G.192.

(2006a): Los jóvenes y el empleo en América Latina: desafíos y perspectivas ante el nuevo escenario laboral, Bogotá, D.C., Comisión Económica para América Latina y el Caribe (CEPAL)/ Mayol ediciones.

(2006b): Tendencias recientes de la inserción de los jóvenes latinoamericanos en el mercado laboral, en J. Weller, Los jóvenes y el empleo en América Latina: Desafios y perspectivas ante el nuevo escenario laboral, Bogotá, D.C., Comisión Económica para América Latina y el Caribe (CEPAL)/Mayol ediciones. 